\title{
A Chevalley formula for the equivariant quantum $K$-theory of cominuscule varieties
}

\author{
Anders S. Buch, Pierre-Emmanuel Chaput, Leonardo C. Mihalcea and \\ Nicolas Perrin
}

\begin{abstract}
We prove a type-uniform Chevalley formula for multiplication with divisor classes in the equivariant quantum $K$-theory ring of any cominuscule flag variety $G / P$. We also prove that multiplication with divisor classes determines the equivariant quantum $K$ theory of arbitrary flag varieties. These results prove a conjecture of Gorbounov and Korff concerning the equivariant quantum $K$-theory of Grassmannians of Lie type A.
\end{abstract}

\section{Introduction}

Let $X=G / P$ be a flag variety defined by a semisimple complex Lie group $G$ and a parabolic subgroup $P$. The (small) equivariant quantum $K$-theory ring $\mathrm{QK}_{T}(X)$ of Givental [Giv00] is a common generalization of the main cohomology theories considered in Schubert calculus, including $K$-theory, equivariant cohomology, and quantum cohomology. Equivariant quantum $K$ theory is the most general theory for which the associated Schubert structure constants have positivity properties that are either known [Gra01, Mih06b, Bri02, AGM11, AC15] or conjectured [LM06, LP07, BM11]. In this paper, we prove a Chevalley formula that combinatorially determines the ring $\mathrm{QK}_{T}(X)$ when $X$ is a cominuscule variety, that is, a Grassmann variety $\operatorname{Gr}(m, n)$ of type A, a Lagrangian Grassmannian LG $(n, 2 n)$, a maximal orthogonal Grassmannian $\mathrm{OG}(n, 2 n)$, a quadric hypersurface $Q^{n}$, or one of two exceptional varieties called the Cayley plane $E_{6} / P_{6}$ and the Freudenthal variety $E_{7} / P_{7}$.

The Schubert structure constants of the ring $\mathrm{QK}_{T}(X)$ are defined as polynomial expressions in the (equivariant, $K$-theoretic) Gromov-Witten invariants of $X$. The non-equivariant GromovWitten invariants used to define the quantum $K$-theory ring $\mathrm{QK}(X)$ can be computed for some spaces using the reconstruction theorem of Lee and Pandharipande [LP04] together with the $K$-theoretic $J$-function of Taipale [Tai13]. However, infinitely many Gromov-Witten invariants are required to evaluate the product of two Schubert classes in $\mathrm{QK}(X)$, and as a result we do not

Received 29 April 2016, accepted in final form 5 June 2017.

2010 Mathematics Subject Classification 14N35 (primary), 19E08, 14N15, 14M15 (secondary).

Keywords: quantum $K$-theory, Chevalley formula, Gromov-Witten invariants, Schubert structure constants, cominuscule flag varieties, Molev-Sagan equations.

This journal is (C) Foundation Compositio Mathematica 2018. This article is distributed with Open Access under the terms of the Creative Commons Attribution Non-Commercial License, which permits non-commercial reuse, distribution, and reproduction in any medium, provided that the original work is properly cited. For commercial re-use, please contact the Foundation Compositio Mathematica.

The first author was supported in part by NSF grants DMS-1205351 and DMS-1503662.

The third author was supported in part by NSA grants H98230-13-1-0208 and H98320-16-1-0013, and a Simons Collaboration Grant.

The fourth author was supported by a public grant as part of the Investissement d'avenir project, reference ANR-11-LABX-0056-LMH, LabEx LMH 


\section{A Chevalley formula for equivariant quantum $K$-Theory}

know much about the structure of this ring for general flag varieties. Iritani, Milanov, and Tonita have recently used an enhanced reconstruction theorem to compute the quantum $K$-theory of the variety $\mathrm{SL}(3) / B$ of complete flags in $\mathbb{C}^{3}[\mathrm{IMT} 15]$.

When $X$ is a Grassmann variety of type $\mathrm{A}$, the non-equivariant ring $\mathrm{QK}(X)$ is determined by a Pieri formula of Buch and Mihalcea for products that involve special Schubert classes [BM11]. More generally, when the Picard group of $X$ has rank 1, it has been proved by the authors of the present paper that all products of Schubert classes in the equivariant ring $\mathrm{QK}_{T}(X)$ contain only finitely many non-zero terms [BCMP13, BCMP16]. As a consequence, the multiplication table of $\mathrm{QK}_{T}(X)$ can be determined from finitely many Gromov-Witten invariants.

Our main result is a type-uniform Chevalley formula that describes the product of an arbitrary Schubert class with the divisor class in $\mathrm{QK}_{T}(X)$ when $X$ is any cominuscule variety. The Schubert classes on a cominuscule variety can be indexed by diagrams of boxes that generalize the Young diagrams known from the Schubert calculus of classical Grassmannians. Our Chevalley formula can be formulated in terms of adding or removing boxes from these diagrams, and can equivalently be formulated in terms of operations on Weyl group elements. Chevalley formulas have previously been proved for the equivariant $K$-theory ring $K^{T}(X)$ by Lenart and Postnikov [LP07], and for the equivariant quantum cohomology ring $\mathrm{QH}_{T}(X)$ by Mihalcea [Mih07]; these formulas are valid for arbitrary flag varieties.

The Gromov-Witten invariants of any cominuscule variety $X$ can be computed using the 'quantum-equals-classical' theorem [BKT03, CMP08, BM11, CP11b], which states that any (three-point, genus zero) Gromov-Witten invariant of $X$ is equal to a $K$-theoretic triple intersection on a related space. When $X$ is a Grassmannian of type A, this related space is a two-step partial flag variety of kernel-span pairs [Buc03]. Our Chevalley formula is proved using an alternative version of the quantum-equals-classical theorem, which expresses the GromovWitten invariants of $X$ in terms of projected Gromov-Witten varieties [KLS13, BCMP18]. Here, a projected Gromov-Witten variety is the closure of the union of all rational curves of a fixed degree that pass through two opposite Schubert varieties in $X$. Our proof shows that the quantum terms in the Chevalley formula can be obtained by applying a linear operator to the classical terms. We also provide an alternative geometric proof of Lenart and Postnikov's Chevalley formula [LP07], specialized to the equivariant $K$-theory of cominuscule varieties. This proof is type uniform for all minuscule varieties, but requires specialized arguments for Lagrangian Grassmannians and quadrics of odd dimension. It would be interesting to investigate whether similar geometric arguments extend beyond the case of cominuscule flag varieties.

Gorbounov and Korff have recently used ideas from integrable systems to define an algebra $q h^{*}(\operatorname{Gr}(m, n))$ that they conjecture is isomorphic to the equivariant quantum $K$-theory ring $\mathrm{QK}_{T}(\mathrm{Gr}(m, n))$ of a Grassmannian of type A [GK17]. In our last section, we use Molev-Sagan equations [MS99, KT03] to prove, for any flag variety $X$, that the structure of the ring $\mathrm{QK}_{T}(X)$, including its Schubert structure constants and the underlying Gromov-Witten invariants of $X$, is uniquely determined by products involving divisors. Since Gorbounov and Korff prove that their algebra $q h^{*}(\operatorname{Gr}(m, n))$ satisfies the same Chevalley formula as the one proved for equivariant quantum $K$-theory in this paper, we obtain a proof of their conjecture. In particular, a presentation and a Giambelli formula from [GK17] for the algebra $q h^{*}(\operatorname{Gr}(m, n))$ are also valid in equivariant quantum $K$-theory.

Our paper is organized as follows. In Section 2, we recall the definition of quantum $K$-theory for arbitrary flag varieties. Section 3 covers quantum $K$-theory of cominuscule varieties and proves the quantum part of our Chevalley formula. Two equivalent versions of this formula are 


\section{A.S. Buch, P.-E. Chaput, L.C. Mihalcea And N. Perrin}

given in Theorem 3.9 and Corollary 3.10, which are followed by examples. Section 4 contains our geometric proof of the Chevalley formula for the equivariant $K$-theory of cominuscule varieties. Finally, Section 5 proves that the equivariant quantum $K$-theory of any flag variety is uniquely determined by products with divisor classes; see Proposition 5.13.

\section{The quantum $K$-theory of flag varieties}

\subsection{Equivariant $K$-theory}

All algebraic varieties and schemes in this paper will be defined over the complex numbers $\mathbb{C}$. Given a variety $X$ with an action of an algebraic torus $T=\left(\mathbb{C}^{*}\right)^{n}$, we let $K_{T}(X)$ denote the Grothendieck group of $T$-equivariant coherent $\mathcal{O}_{X}$-modules. This group is a module over the Grothendieck ring $K^{T}(X)$ of $T$-equivariant vector bundles over $X$. If $X$ is non-singular, then the implicit map $K^{T}(X) \rightarrow K_{T}(X)$ that sends a vector bundle to its sheaf of sections is an isomorphism. Our main references for equivariant $K$-theory are [CG97, Chapter 5] and [Ful98, $\S 15.1]$. A shorter summary of the main properties can also be found in [BM11, §3]. Pullback along the structure morphism $X \rightarrow$ \{point gives $K^{T}(X)$ a structure of algebra over the ring $\Gamma=K^{T}$ (point). This ring $\Gamma$ is simply the ring of virtual representations of $T$. Given any character $\alpha: T \rightarrow \mathbb{C}^{*}$, we let $\mathbb{C}_{\alpha}$ denote the 1-dimensional representation of $T$ defined by $t . z=\alpha(t) z$ for $t \in T$ and $z \in \mathbb{C}_{\alpha}$. The ring $\Gamma$ has a $\mathbb{Z}$-basis consisting of the classes $\left[\mathbb{C}_{\alpha}\right]$ of these representations. When $X$ is projective, we let $\chi_{X}: K_{T}(X) \rightarrow \Gamma$ denote the pushforward along the structure morphism of $X$. This is a homomorphism of $\Gamma$-modules by the projection formula.

\subsection{Schubert varieties}

Let $X=G / P$ be a flag variety defined by a complex semisimple linear algebraic group $G$ and a parabolic subgroup $P$. Fix a maximal torus $T$ and a Borel subgroup $B$ such that $T \subset B \subset P \subset$ $G$, and let $B^{-} \subset G$ be the opposite Borel subgroup defined by $B \cap B^{-}=T$. Let $W=N_{G}(T) / T$ be the Weyl group of $G$, let $W_{P}=N_{P}(T) / T$ be the Weyl group of $P$, and let $W^{P} \subset W$ be the set of minimal representatives of the cosets in $W / W_{P}$. Each element $w \in W$ defines a $B$-stable Schubert variety $X_{w}=\overline{B w \cdot P}$ and an (opposite) $B^{-}$-stable Schubert variety $X^{w}=\overline{B^{-} w \cdot P}$ in $X$. When $w \in W^{P}$ is a minimal representative, we have $\operatorname{dim}\left(X_{w}\right)=\operatorname{codim}\left(X^{w}, X\right)=\ell(w)$, where $\ell(w)$ denotes the length of $w$. A product $w=u_{1} u_{2} \cdot \ldots \cdot u_{m}$ of elements in $W$ will be called reduced if $\ell(w)=\sum \ell\left(u_{i}\right)$. This implies that any consecutive subproduct $u_{i} u_{i+1} \cdot \ldots \cdot u_{j}$ is also reduced. The dual element of $u \in W^{P}$ is $u^{\vee}=w_{0} u w_{P}$, where $w_{0}$ is the longest element in $W$ and $w_{P}$ is the longest element in $W_{P}$. Notice that $1^{\vee}$ is the longest element in $W^{P}$. Let $\Phi$ be the root system of $(G, T)$, interpreted as a set of characters of $T$. Let $\Phi^{+}$be the set of positive roots determined by $B$, let $\Delta \subset \Phi^{+}$be the set of simple roots, and let $\Delta_{P} \subset \Delta$ be the set of simple roots $\beta$ for which the associated reflection $s_{\beta}$ belongs to $W_{P}$. We need the following topological fact.

Lemma 2.1. Let $Z \subset X$ be a $T$-stable closed subvariety, and let $x_{0} \in X^{T}$ be a $T$-fixed point such that $Z \cap B . x_{0} \neq \emptyset$. Then $x_{0} \in Z$.

Proof. Choose any group homomorphism $\phi: \mathbb{C}^{*} \rightarrow T$ such that for each $\alpha \in \Phi^{+}$, the composition $\alpha \phi: \mathbb{C}^{*} \rightarrow \mathbb{C}^{*}$ is given by $\alpha(\phi(s))=s^{m}$ for some $m>0$. Let $U_{\alpha} \subset G$ be the $T$-stable subgroup with $\operatorname{Lie}\left(U_{\alpha}\right)=\operatorname{Lie}(G)_{\alpha}$. It follows from [Hum75, Theorem 26.3] that $\lim _{s \rightarrow 0} \phi(s) b \phi(s)^{-1}=1$ for any element $b \in U_{\alpha}, \alpha \in \Phi^{+}$. Since $B$ is generated by $T$ and the subgroups $U_{\alpha}$ for $\alpha \in \Phi^{+}$, we 


\section{A Chevalley formula for equivariant quantum $K$-Theory}

deduce that $\lim _{s \rightarrow 0} \phi(s) b \phi(s)^{-1} \in T$ for any $b \in B$. Now, choose $b \in B$ such that $b . x_{0} \in Z$. We then obtain $x_{0}=\left(\lim _{s \rightarrow 0} \phi(s) b \phi(s)^{-1}\right) \cdot x_{0}=\lim _{s \rightarrow 0} \phi(s) b . x_{0} \in Z$, as required.

\subsection{Schubert structure constants}

The equivariant $K$-theory ring $K^{T}(X)$ of the flag variety $X$ has a basis over $\Gamma$ consisting of the (opposite) Schubert classes $\mathcal{O}^{w}=\left[\mathcal{O}_{X^{w}}\right]$ for $w \in W^{P}$. The classes $\mathcal{O}_{w}=\left[\mathcal{O}_{X_{w}}\right]$ form an alternative basis. The Schubert structure constants of $K^{T}(X)$ are the classes $N_{u, v}^{w, 0} \in \Gamma$ defined for $u, v, w \in W^{P}$ by the identity

$$
\mathcal{O}^{u} \cdot \mathcal{O}^{v}=\sum_{w} N_{u, v}^{w, 0} \mathcal{O}^{w}
$$

Let $\mathcal{O}_{w}^{\vee} \in K^{T}(X)$ denote the basis element dual to $\mathcal{O}^{w}$, defined by $\chi_{X}\left(\mathcal{O}^{u} \cdot \mathcal{O}_{w}^{\vee}\right)=\delta_{u, w}$ for $u, w \in W^{P}$. We then have $N_{u, v}^{w, 0}=\chi_{X}\left(\mathcal{O}^{u} \cdot \mathcal{O}^{v} \cdot \mathcal{O}_{w}^{\vee}\right)$. The boundary of the Schubert variety $X_{w}$ is the closed subvariety defined by $\partial X_{w}=X_{w} \backslash B w \cdot P$. Brion has proved the identity

$$
\mathcal{O}_{w}^{\vee}=\left[I_{\partial X_{w}}\right] \text {, }
$$

where $I_{\partial X_{w}} \subset \mathcal{O}_{X_{w}}$ denotes the ideal sheaf of this boundary [Bri02].

Calculations in the ring $K^{T}(X)$ may be carried out by utilizing that restriction to the set of $T$-fixed points $X^{T}=\left\{w . P \mid w \in W^{P}\right\}$ provides an injective ring homomorphism $K^{T}(X) \rightarrow K^{T}\left(X^{T}\right)=\prod_{w \in W^{P}} \Gamma$ [KK90] (see also [GKM98]). The images of Schubert classes under this map are given by the restriction formulas in [AJS94, Bil97, GK15, Wil06] (see also [Knu08]). The ring structure of $K^{T}(X)$ is also determined by the Chevalley formula of Lenart and Postnikov [LP07], which provides an explicit expression for the product of any Schubert class with a divisor in $K^{T}(X)$. A version of this formula for cominuscule varieties will be discussed in Section 3.4. Other useful formulas for structure constants in various special cases can be found in, for example, [BS16, Knu10, Buc15, PY17] and the references therein.

\subsection{Quantum $K$-theory}

A homology class $d=\sum d_{\beta}\left[X_{s_{\beta}}\right] \in H_{2}(X ; \mathbb{Z})$, with the sum over $\beta \in \Delta \backslash \Delta_{P}$, is called an effective degree if $d_{\beta} \geqslant 0$ for each $\beta$. For $d, e \in H_{2}(X ; \mathbb{Z})$, we write $e \leqslant d$ if and only if $d-e$ is effective. For any effective degree $d$, we let $\overline{\mathcal{M}}_{0, n}(X, d)$ denote the Kontsevich moduli space of $n$-pointed stable maps to $X$ of genus zero and degree $d$ [FP97]. This space is equipped with evaluation maps $\mathrm{ev}_{i}: \overline{\mathcal{M}}_{0, n}(X, d) \rightarrow X$ for $1 \leqslant i \leqslant n$. Given classes $\sigma_{1}, \sigma_{2}, \ldots, \sigma_{n} \in K^{T}(X)$, define a corresponding (equivariant $K$-theoretic) Gromov-Witten invariant of $X$ by

$$
I_{d}\left(\sigma_{1}, \sigma_{2}, \ldots, \sigma_{n}\right)=\chi_{\overline{\mathcal{M}}_{0, n}(X, d)}\left(\operatorname{ev}_{1}^{*}\left(\sigma_{1}\right) \cdot \operatorname{ev}_{2}^{*}\left(\sigma_{2}\right) \cdot \ldots \cdot \mathrm{ev}_{n}^{*}\left(\sigma_{n}\right)\right) \in \Gamma .
$$

This invariant is $\Gamma$-linear in each argument $\sigma_{i}$.

The (small) $T$-equivariant quantum $K$-theory ring of $X$ is an algebra $\mathrm{QK}_{T}(X)$ over the ring of formal power series $\Gamma \llbracket q \rrbracket=\Gamma \llbracket q_{\beta} \mid \beta \in \Delta \backslash \Delta_{P} \rrbracket$, which has one variable $q_{\beta}$ for each simple root $\beta \in \Delta \backslash \Delta_{P}$. We have $\mathrm{QK}_{T}(X)=K^{T}(X) \otimes_{\Gamma} \Gamma \llbracket q \rrbracket$ as a module over $\Gamma \llbracket q \rrbracket$. In other words, $\mathrm{QK}_{T}(X)$ is a free module over $\Gamma \llbracket q \rrbracket$ with a basis consisting of the Schubert classes $\mathcal{O}^{w}$ for $w \in W^{P}$. For $d=\sum d_{\beta}\left[X_{s_{\beta}}\right]$, we write $q^{d}=\prod_{\beta} q_{\beta}^{d_{\beta}}$. Multiplication in $\mathrm{QK}_{T}(X)$ is defined by

$$
\mathcal{O}^{u} \star \mathcal{O}^{v}=\sum_{w, d \geqslant 0} N_{u, v}^{w, d} q^{d} \mathcal{O}^{w}
$$

where the sum is over all effective degrees $d$ and $w \in W^{P}$. The structure constants $N_{u, v}^{w, d}$ are 


\section{A.S. Buch, P.-E. Chaput, L.C. Mihalcea And N. Perrin}

defined recursively by

$$
N_{u, v}^{w, d}=I_{d}\left(\mathcal{O}^{u}, \mathcal{O}^{v}, \mathcal{O}_{w}^{\vee}\right)-\sum_{\kappa, 0<e \leqslant d} N_{u, v}^{\kappa, d-e} I_{e}\left(\mathcal{O}^{\kappa}, \mathcal{O}_{w}^{\vee}\right)
$$

where this sum is over all $\kappa \in W^{P}$ and degrees $e$ for which $0<e \leqslant d$. A theorem of Givental states that this defines an associative product [Giv00] (see also [Wit95, RT94, KM94, Lee04]).

The ring $\mathrm{QK}_{T}(X)$ is a formal deformation of the equivariant $K$-theory ring $K^{T}(X)$. It is best understood when $X$ is a cominuscule variety. This case will be discussed in Section 3 . When the Picard group of $X$ has rank 1, it has been proved in [BCMP13, BCMP16] that the product $\mathcal{O}^{u} \star \mathcal{O}^{v}$ contains only finitely many non-zero terms. It is an open question if this holds for general flag varieties. Combinatorial models for the equivariant quantum $K$-theory of complete flag manifolds $G / B$ have been defined by Lenart and Maeno [LM06] and by Lenart and Postnikov [LP07]. These models are correct for $\mathbb{P}^{1}$ [BM11] and consistent with the computation of the quantum $K$-theory of $\mathrm{SL}(3) / B$ [IMT15], but for all other spaces it is an open problem to prove that the models agree with the geometric definition of $\mathrm{QK}_{T}(X)$. In addition, the lack of functoriality of quantum $K$-theory makes it unclear how these models relate to cominuscule varieties. Another model for the equivariant quantum $K$-theory of Grassmannians of type A has been conjectured by Gorbounov and Korff [GK17]. This model is a consequence of the results proved in this paper (see Remark 5.15). Finally, it is expected that the structure constants of $\mathrm{QK}_{T}(X)$ satisfy the following positivity property [GR04, LM06, LP07, BM11].

Conjecture 2.2. Let $u, v, w \in W^{P}$, let $d \in H_{2}(X ; \mathbb{Z})$ be an effective degree, and set $s=$ $\ell(u)+\ell(v)+\ell(w)+\int_{d} c_{1}\left(T_{X}\right)$. Then we have

$$
(-1)^{s} N_{u, v}^{w, d} \in \mathbb{N}\left[\left[\mathbb{C}_{-\beta}\right]-1 \mid \beta \in \Delta\right] .
$$

In other words, the structure constant $N_{u, v}^{w, d}$ can be written, up to a sign, as a polynomial with non-negative integer coefficients in the classes $\left[\mathbb{C}_{-\beta}\right]-1$ for $\beta \in \Delta$. This has been proved for the structure constants $N_{u, v}^{w, 0}$ of $K^{T}(X)$ by Anderson, Griffeth, and Miller [AGM11]. Earlier, it was proved by Brion [Bri02] that the structure constants of the ordinary $K$-theory ring $K(X)$ have signs that alternate with codimension.

\subsection{Curve neighborhoods}

Given a closed subvariety $\Omega \subset X$ and an effective degree $d \in H_{2}(X ; \mathbb{Z})$, we let $\Gamma_{d}(\Omega)$ denote the closure of the union of all rational curves of degree $d$ in $X$ that pass through $\Omega$. Equivalently, we have $\Gamma_{d}(\Omega)=\mathrm{ev}_{2}\left(\mathrm{ev}_{1}^{-1}(\Omega)\right)$, where $\mathrm{ev}_{1}, \mathrm{ev}_{2}: \overline{\mathcal{M}}_{0,2}(X, d) \rightarrow X$ are the evaluation maps. It was proved in [BCMP13] that $\Gamma_{d}(\Omega)$ is irreducible whenever $\Omega$ is irreducible. In particular, if $\Omega$ is a $B$-stable Schubert variety in $X$, then so is $\Gamma_{d}(\Omega)$. Given $w \in W^{P}$, we may therefore define related Weyl group elements $w(d)$ and $w(-d)$ in $W^{P}$ by the identities $\Gamma_{d}\left(X_{w}\right)=X_{w(d)}$ and $\Gamma_{d}\left(X^{w}\right)=X^{w(-d)}$. We have $w(-d) \leqslant w \leqslant w(d)$ in the Bruhat order of $W^{P}$, and since $X^{w}$ is a translate of $X_{w^{\vee}}$, we obtain $w(-d)^{\vee}=w^{\vee}(d)$. A combinatorial description of $w(d)$ and $w(-d)$ can be found in [BCMP13] or Section 3.2 when $X$ is cominuscule and in [BM15] for general flag varieties.

It was proved in [BCMP13] that $I_{d}\left(\mathcal{O}^{w}\right)=1$; that is, any single-pointed Gromov-Witten invariant of a Schubert class is equal to 1. More generally, it follows from [BCMP13, Proposition 3.2] that two-pointed Gromov-Witten invariants are given by the identity

$$
I_{d}\left(\mathcal{O}^{u}, \mathcal{O}_{w}^{\vee}\right)=\chi_{X}\left(\mathcal{O}^{u(-d)} \cdot \mathcal{O}_{w}^{\vee}\right)=\delta_{u(-d), w} \cdot
$$




\section{A Chevalley formula for equivariant quantum $K$-theory}

As a consequence, the definition (2.2) of the structure constants of $\mathrm{QK}_{T}(X)$ is equivalent to the identity

$$
I_{d}\left(\mathcal{O}^{u}, \mathcal{O}^{v}, \mathcal{O}_{w}^{\vee}\right)=\sum_{0 \leqslant e \leqslant d \kappa: \kappa(-e)=w} \sum_{u, v}^{\kappa, d-e}
$$

For any classes $\sigma_{1}, \sigma_{2} \in K^{T}(X)$, we define the power series

$$
\sigma_{1} \odot \sigma_{2}=\sum_{w, d \geqslant 0} I_{d}\left(\sigma_{1}, \sigma_{2}, \mathcal{O}_{w}^{\vee}\right) q^{d} \mathcal{O}^{w}
$$

in $\mathrm{QK}_{T}(X)$. We also define an automorphism $\Psi: \mathrm{QK}_{T}(X) \rightarrow \mathrm{QK}_{T}(X)$ of $\Gamma \llbracket q \rrbracket$-modules by

$$
\Psi\left(\mathcal{O}^{w}\right)=\sum_{e \geqslant 0} q^{e} \mathcal{O}^{w(-e)}
$$

Equation (2.3) is equivalent to the following statement.

Proposition 2.3. For $\sigma_{1}, \sigma_{2} \in K^{T}(X)$, we have $\sigma_{1} \odot \sigma_{2}=\Psi\left(\sigma_{1} \star \sigma_{2}\right)$ in $\mathrm{QK}_{T}(X)$.

\section{Cominuscule varieties}

\subsection{The Bruhat order}

A simple root $\gamma \in \Delta$ is called cominuscule if, when the highest root is written as a linear combination of simple roots, the coefficient of $\gamma$ is 1 . The flag variety $X=G / P$ is called cominuscule if $\Delta \backslash \Delta_{P}$ consists of a single cominuscule root $\gamma$. If, in addition, the root system $\Phi$ is simply laced, then $X$ is also called minuscule. This holds for all the cominuscule varieties listed in the introduction, except for Lagrangian Grassmannians and quadrics of odd dimension. We will assume that $X$ is cominuscule in this section. In this case, it was proved by Proctor that the Bruhat order on $W^{P}$ is a distributive lattice that agrees with the left weak Bruhat order [Pro84]. Stembridge has proved that all elements of $W^{P}$ are fully commutative, which means that any reduced expression for an element of $W^{P}$ can be obtained from any other by interchanging commuting simple reflections. We proceed to summarize the facts we need in more detail. Proofs of our claims can be found in [Pro84, Ste96, Per07, BS16].

The root lattice $\operatorname{Span}_{\mathbb{Z}}(\Delta)$ has a partial order defined by $\alpha^{\prime} \leqslant \alpha$ if and only if $\alpha-\alpha^{\prime}$ can be written as a sum of positive roots. Let $\mathcal{P}_{X}=\{\alpha \in \Phi \mid \alpha \geqslant \gamma\}$ be the set of positive roots $\alpha$ for which the coefficient of the cominuscule root $\gamma$ is 1 , with the induced partial order. For any element $u \in W$, we let $I(u)=\left\{\alpha \in \Phi^{+} \mid u . \alpha<0\right\}$ denote the inversion set of $u$. We then have $\ell(u)=|I(u)|$. The assignment $u \mapsto I(u)$ restricts to a bijection between the elements of $W^{P}$ and the lower-order ideals of $\mathcal{P}_{X}$. This assignment is order preserving in the sense that $u \leqslant v$ if and only if $I(u) \subset I(v)$. Given a lower-order ideal $\lambda \subset \mathcal{P}_{X}$, let $\lambda=\left\{\alpha_{1}, \alpha_{2}, \ldots, \alpha_{|\lambda|}\right\}$ be any ordering of its elements that is compatible with the partial order $\leqslant$; that is, $\alpha_{i}<\alpha_{j}$ implies $i<j$. Then the element of $W^{P}$ corresponding to $\lambda$ is the product of reflections $w_{\lambda}=s_{\alpha_{1}} s_{\alpha_{2}} \cdot \ldots \cdot s_{\alpha_{|\lambda|}}$. The order ideal $\lambda$ is a generalization of the Young diagrams and shifted Young diagrams known from the Schubert calculus of the classical Grassmannians. For this reason, the roots in $\mathcal{P}_{X}$ will sometimes be called boxes, and the order ideal $I(u)$ will be called the shape of $u$.

Given two elements $u, w \in W^{P}$ with $u \leqslant w$, we will use the notation $w / u=w u^{-1} \in W$. Since the Bruhat order on $W^{P}$ agrees with the left weak Bruhat order, we have $\ell(w / u)=\ell(w)-\ell(u)$. For any root $\alpha \in \mathcal{P}_{X}$, consider the order ideal $\lambda=\left\{\alpha^{\prime} \in \mathcal{P}_{X} \mid \alpha^{\prime}<\alpha\right\}$ of roots that are smaller than $\alpha$, and set $\delta(\alpha)=w_{\lambda}$. $\alpha$. Then $s_{\delta(\alpha)}=w_{\lambda} s_{\alpha} w_{\lambda}^{-1}=w_{\lambda \cup \alpha} / w_{\lambda}$ has length 1 . It follows that 


\section{A.S. Buch, P.-E. Chaput, L.C. Mihalcea and N. Perrin}

$\delta: \mathcal{P}_{X} \rightarrow \Delta$ is a labeling of the boxes in $\mathcal{P}_{X}$ by simple roots. In addition, the element $w / u$ depends only on the skew shape $I(w) \backslash I(u)$. More precisely, if $I(w) \backslash I(u)=\left\{\alpha_{1}, \alpha_{2}, \ldots, \alpha_{\ell}\right\}$ is any ordering compatible with $\leqslant$, then $w / u=s_{\delta\left(\alpha_{\ell}\right)} \cdots s_{\delta\left(\alpha_{2}\right)} s_{\delta\left(\alpha_{1}\right)}$ is a reduced expression for $w / u$. In the special case $u=1$, every reduced expression for $w$ can be obtained in this way.

We will say that $w / u$ is a rook strip if this element of $W$ is a product of commuting simple reflections. Equivalently, no two roots in $I(w) \backslash I(u)$ are comparable by the order $\leqslant$. We call $w / u$ a short rook strip if it is a product of commuting reflections defined by short simple roots; that is, $I(w) \backslash I(u)$ consists of incomparable short roots. Notice that if the root system $\Phi$ is simply laced, then all roots are long by convention, so $w / u$ is a short rook strip if and only if $w=u$.

The Bruhat order on $W^{P}$ is a distributive lattice with operations $u \cap v$ and $u \cup v$ for $u, v \in W^{P}$. These operations are defined combinatorially by $I(u \cap v)=I(u) \cap I(v)$ and $I(u \cup v)=I(u) \cup I(v)$, and geometrically by $X_{u \cap v}=X_{u} \cap X_{v}$ and $X^{u \cup v}=X^{u} \cap X^{v}$. Notice that for any $u, z \in W^{P}$, we have $(u \cup z) / z=u /(u \cap z)$. Since dualization of Weyl group elements is an order-reversing involution of $W^{P}$, we also have $(u \cap v)^{\vee}=u^{\vee} \cup v^{\vee}$ and $(u \cup v)^{\vee}=u^{\vee} \cap v^{\vee}$.

\subsection{Curve neighborhoods}

Since $P$ is a maximal parabolic subgroup of $G$, it follows that $H_{2}(X ; \mathbb{Z})=\mathbb{Z}$. For $u \in W^{P}$ and $d \in \mathbb{N}$, we let $u(d), u(-d) \in W^{P}$ be the unique Weyl group elements for which $\Gamma_{d}\left(X_{u}\right)=X_{u(d)}$ and $\Gamma_{d}\left(X^{u}\right)=X^{u(-d)}$. Since $X$ is cominuscule, we have $u(d)\left(d^{\prime}\right)=u\left(d+d^{\prime}\right)$ and $u(-d)\left(-d^{\prime}\right)=$ $u\left(-d-d^{\prime}\right)$ for any effective degrees $d, d^{\prime} \in \mathbb{N}$; see [CMP08] or [BCMP13, Lemma 4.2]. However, it is easy to find examples where both $u\left(d^{\prime}\right)(-d)$ and $u(-d)\left(d^{\prime}\right)$ are different from $u\left(d^{\prime}-d\right)$. It was proved in [BCMP13, Lemma 4.4] that $u(1)$ is the minimal representative of the coset $u w_{P} s_{\gamma} W_{P}$ whenever $X_{u} \neq X$. Equivalently, $u(-1)$ is the minimal representative of $u s_{\gamma} W_{P}$ whenever $u \neq 1$.

For $d \geqslant 0$, we let $z_{d} \in W^{P}$ be the unique element such that $X_{z_{d}}=\Gamma_{d}(1 . P)$. This element satisfies that $z_{d} w_{P}$ is inverse to itself, where $w_{P}$ denotes the longest element in $W_{P}$. In fact, if we let $C \subset X$ be any stable curve of degree $d$ from 1.P to $z_{d} . P$, then $\left(z_{d} w_{P}\right)^{-1}$. $C$ is a curve of the same degree from 1.P to $\left(z_{d} w_{P}\right)^{-1} . P$, so we obtain $\left(z_{d} w_{P}\right)^{-1} . P \in \Gamma_{d}(1 . P)$. This implies that $\left(z_{d} w_{P}\right)^{-1} \leqslant z_{d} w_{P}$ and therefore $\left(z_{d} w_{P}\right)^{-1}=z_{d} w_{P}$. It follows that $z_{d} w_{P}$ is the maximal representative of the left coset $W_{P} z_{d} w_{P}$. If $X_{z_{d}} \neq X$, we deduce that $\mathcal{P}_{X} \backslash I\left(z_{d}\right)$ contains a unique minimal box $\alpha_{d+1}$, and this box satisfies $\delta\left(\alpha_{d+1}\right)=\gamma$. In other words, we have $I\left(z_{d}\right)=$ $\left\{\alpha \in \mathcal{P}_{X} \mid \alpha \ngtr \alpha_{d+1}\right\}$. Notice also that since $z_{d+1}=z_{d}(1)$ is the minimal representative of the coset $z_{d} w_{P} s_{\gamma} W_{P}$, we may choose $x \in W_{P}$ such that $z_{d+1} w_{P}=z_{d} w_{P} s_{\gamma} x^{-1}$ holds as an identity of reduced products. This implies that $z_{d+1} w_{P}=\left(z_{d+1} w_{P}\right)^{-1}=x s_{\gamma}\left(z_{d} w_{P}\right)^{-1}=x s_{\gamma} z_{d} w_{P}$; hence, $z_{d+1}=x s_{\gamma} z_{d}$ expresses $z_{d+1}$ as a reduced product. Since every reduced expression of $z_{d+1}$ corresponds to an ordering of the boxes of $I\left(z_{d+1}\right)$, this implies that $\alpha_{d+1}$ is the only box of $I\left(z_{d+1}\right) \backslash I\left(z_{d}\right)$ that is sent to $\gamma$ by the map $\delta$. In fact, we have $\delta^{-1}(\gamma)=\left\{\alpha_{1}<\alpha_{2}<\cdots<\alpha_{d_{X}(2)}\right\}$, where $d_{X}(2)$ is the smallest degree of a rational curve joining two general points in $X$.

Lemma 3.1. For $u \in W^{P}$ and $d \in \mathbb{N}$, we have $u(-d)=u /\left(u \cap z_{d}\right)=\left(u \cup z_{d}\right) / z_{d}$ and $u(d)=$ $\left(u \cap z_{d}^{\vee}\right) z_{d}$.

Proof. For $d=0$, the lemma follows because $u(0)=u$ and $z_{0}=1$. Let $d>0$, and assume by induction that $u(1-d)=u /\left(u \cap z_{d-1}\right)$. Notice that if $u(1-d)=1$, then the lemma holds for $d$ because $u \leqslant z_{d-1} \leqslant z_{d}$ and $u(-d)=1$. We may therefore assume that $u(1-d) \neq 1$ or, equivalently, $\alpha_{d} \in I(u)$.

Set $v=u /\left(u \cap z_{d}\right)=\left(u \cup z_{d}\right) / z_{d}$. Then we have an identity of reduced products $v z_{d} w_{P}=$ $\left(u \cup z_{d}\right) w_{P}$. Since $z_{d} w_{P}$ is maximal in its left coset $W_{P} z_{d} w_{P}$, it follows that $v$ is minimal in 


\section{A Chevalley formula for equivariant quantum $K$-Theory}

its right coset $v W_{P}$; in other words, $v \in W^{P}$. Since $I\left(u \cap z_{d}\right) \backslash I\left(u \cap z_{d-1}\right) \subset I\left(z_{d}\right) \backslash I\left(z_{d-1}\right)$ and $\alpha_{d} \in I(u)$, it follows that $\left(u \cap z_{d}\right) /\left(u \cap z_{d-1}\right)=y s_{\gamma}$ for some $y \in W_{P}$. Since $u(1-d)=$ $u /\left(u \cap z_{d-1}\right)=v\left(u \cap z_{d}\right) /\left(u \cap z_{d-1}\right)=v y s_{\gamma}$, we obtain $v=u(1-d) s_{\gamma} y^{-1}$. It follows that $v \in W^{P}$ is the minimal representative of the coset $u(1-d) s_{\gamma} W_{P}$, as required.

The last identity now follows because

$$
u^{\vee}(d)=u(-d)^{\vee}=w_{0}\left(u \cup z_{d}\right) z_{d}^{-1} w_{P}=\left(u \cup z_{d}\right)^{\vee}\left(z_{d} w_{P}\right)^{-1} w_{P}=\left(u^{\vee} \cap z_{d}^{\vee}\right) z_{d} .
$$

TABLE 1. Partially ordered sets of cominuscule varieties with $I\left(z_{1}\right)$ highlighted

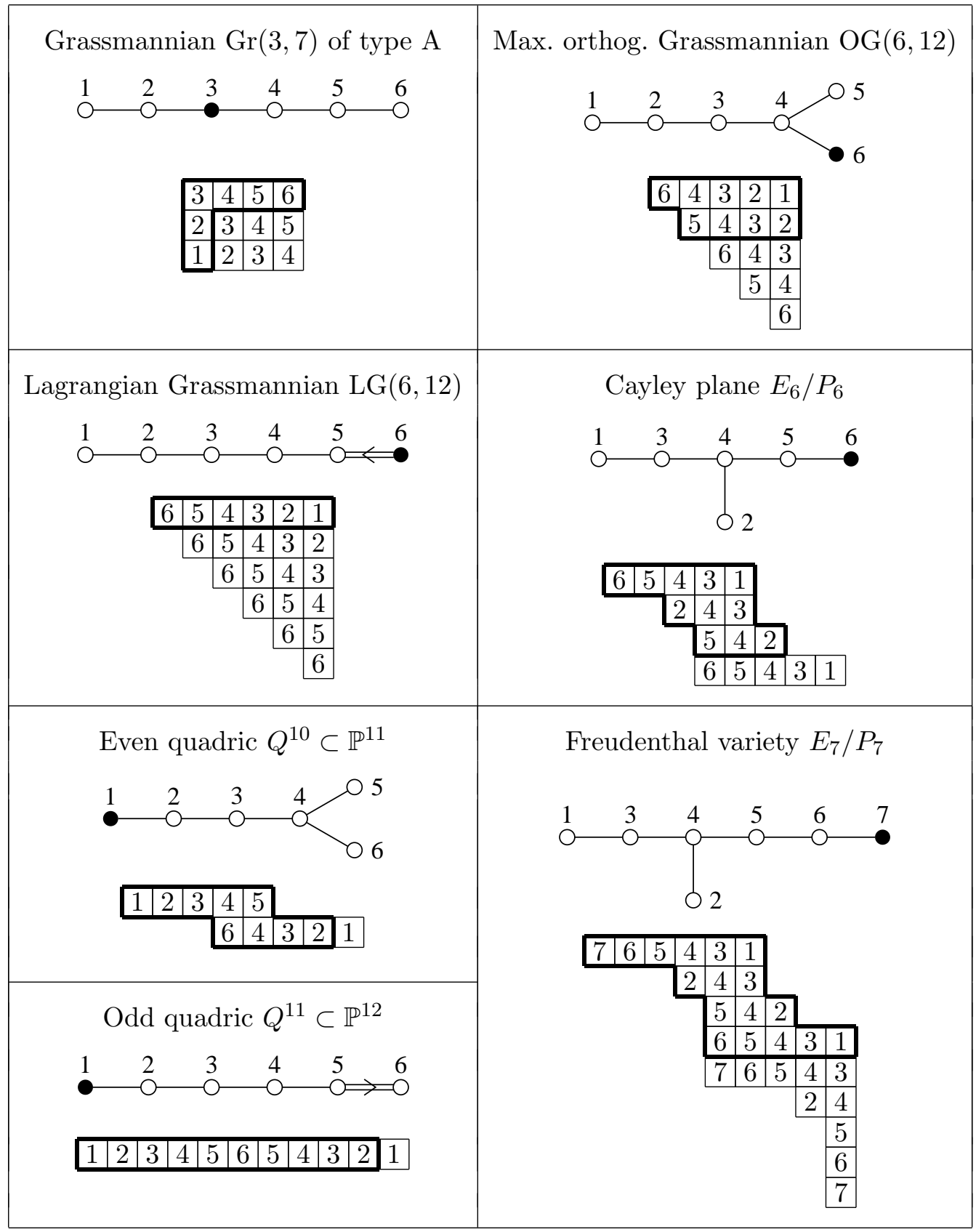

Table 1 displays one cominuscule variety $X$ from each family, together with the associated 


\section{A.S. Buch, P.-E. Chaput, L.C. Mihalcea And N. Perrin}

Dynkin diagram and the partially ordered set $\mathcal{P}_{X}$. The marked node in the Dynkin diagram indicates the cominuscule simple root $\gamma$. The roots of $\mathcal{P}_{X}$ are represented as boxes, and the partial order is given by $\alpha^{\prime} \leqslant \alpha$ if and only if $\alpha^{\prime}$ is located north-west of $\alpha$. Each box $\alpha \in \mathcal{P}_{X}$ is labeled by the number of the simple root $\delta(\alpha)$. In addition, the shape $I\left(z_{1}\right)$ is marked. Lemma 3.1 implies that given any element $u \in W^{P}$, one may obtain the shape of $u(-1)$ from the shape of $u$ by first removing any boxes contained in $I\left(z_{1}\right)$ and then moving the remaining boxes to the upperleft corner of $\mathcal{P}_{X}$. More details about some of the cases can be found in Examples 3.12, 3.13, and Section 4.3.

\subsection{Quantum $K$-theory}

Since $X$ is cominuscule, the equivariant quantum $K$-theory ring $\mathrm{QK}_{T}(X)$ is an algebra over the power series ring $\Gamma \llbracket q \rrbracket$ in a single variable $q_{\gamma}$; we drop the subscript and denote this variable by $q$. We proceed to give a simplified construction of the product in $\mathrm{QK}_{T}(X)$ in the cominuscule case. Let $\psi: K^{T}(X) \rightarrow K^{T}(X)$ be the homomorphism of $\Gamma$-modules defined by $\psi\left(\mathcal{O}^{u}\right)=\mathcal{O}^{u(-1)}$. This map can also be defined geometrically by $\psi=\left(\mathrm{ev}_{2}\right)_{*}\left(\mathrm{ev}_{1}\right)^{*}$, where $\mathrm{ev}_{1}$ and $\mathrm{ev}_{2}$ are the evaluation maps from the moduli space $\overline{\mathcal{M}}_{0,2}(X, 1)$. We extend $\psi$ by linearity to a homomorphism $\psi: \mathrm{QK}_{T}(X) \rightarrow \mathrm{QK}_{T}(X)$ of $\Gamma \llbracket q \rrbracket$-modules.

Proposition 3.2. For $\sigma_{1}, \sigma_{2} \in K^{T}(X)$, we have $\sigma_{1} \star \sigma_{2}=(1-q \psi)\left(\sigma_{1} \odot \sigma_{2}\right)$ in $\operatorname{QK}_{T}(X)$.

Proof. The identity $w(-e-1)=w(-e)(-1)$ among Weyl group elements implies that the operator $\Psi$ from Section 2.5 is given by $\Psi=1+q \psi+q^{2} \psi^{2}+\cdots$. It therefore follows from Proposition 2.3 that $\sigma_{1} \star \sigma_{2}=\Psi^{-1}\left(\sigma_{1} \odot \sigma_{2}\right)=(1-q \psi)\left(\sigma_{1} \odot \sigma_{2}\right)$.

Let $\Gamma_{d}\left(X_{u}, X^{v}\right) \subset X$ denote the union of all stable curves of degree $d$ in $X$ that pass through $X_{u}$ and $X^{v}$. Equivalently, we have $\Gamma_{d}\left(X_{u}, X^{v}\right)=\operatorname{ev}_{3}\left(\operatorname{ev}_{1}^{-1}\left(X_{u}\right) \cap \mathrm{ev}_{2}^{-1}\left(X^{v}\right)\right)$, where $\mathrm{ev}_{1}, \mathrm{ev}_{2}, \mathrm{ev}_{3}: \overline{\mathcal{M}}_{0,3}(X, d) \rightarrow X$ are the evaluation maps. The following version of the quantumequals-classical theorem was proved in [BCMP18, Corollary 4.2].

Theorem 3.3. In $K^{T}(X)$, for $u, v \in W^{P}$, we have

$$
\left[\mathcal{O}_{\Gamma_{d}\left(X_{u}, X^{v}\right)}\right]=\sum_{w \in W^{P}} I_{d}\left(\mathcal{O}_{u}, \mathcal{O}^{v}, \mathcal{O}_{w}^{\vee}\right) \mathcal{O}^{w}
$$

As a consequence, we have $\mathcal{O}_{u} \odot \mathcal{O}^{v}=\sum_{d \geqslant 0}\left[\mathcal{O}_{\Gamma_{d}\left(X_{u}, X^{v}\right)}\right] q^{d}$ for all $u, v \in W^{P}$.

\subsection{A Chevalley formula for $K^{T}(X)$}

Let $J=1-\mathcal{O}^{s_{\gamma}} \in K^{T}(X)$ denote the class of the ideal sheaf of the (opposite) Schubert divisor $X^{s_{\gamma}}$. Our main result is an explicit combinatorial formula for any product of the form $J \star \mathcal{O}^{u}$ in the ring $\mathrm{QK}_{T}(X)$. For $u \in W^{P}$, we let $J_{u}=\left.J\right|_{u . P} \in \Gamma$ denote the restriction of the class $J$ to the $T$-fixed point $u . P \in X$. For $\alpha \in \Phi$, we let $\alpha^{\vee}=(\alpha, \alpha)^{-1} 2 \alpha$ denote the coroot of $\alpha$, and we let $\omega_{\gamma}$ be the fundamental weight corresponding to $\gamma$. The following lemma is proved in Section 4.1.

Lemma 3.4. We have $J_{u}=\left[\mathbb{C}_{u . \omega_{\gamma}-\omega_{\gamma}}\right] \in \Gamma$, and the weight satisfies the identity

$$
\omega_{\gamma}-u \cdot \omega_{\gamma}=\sum_{\alpha \in I(u)}\left(\omega_{\gamma}, \alpha^{\vee}\right) \delta(\alpha) .
$$




\section{A Chevalley formula for equivariant quantum $K$-Theory}

Define a homomorphism $\theta_{0}: K^{T}(X) \rightarrow K^{T}(X)$ of $\Gamma$-modules by

$$
\theta_{0}\left(\mathcal{O}^{u}\right)=\sum_{w / u \text { rook strip }}(-1)^{\ell(w / u)} \mathcal{O}^{w},
$$

where the sum is over all $w \in W^{P}$ for which $u \leqslant w$ and $w / u$ is a rook strip. Equivalently, $\theta_{0}\left(\mathcal{O}^{u}\right)$ is the class of the ideal sheaf of the boundary $\partial X^{u} \subset X^{u}$ by Brion's identity (2.1) together with the following lemma.

Lemma 3.5. The class $\theta_{0}\left(\mathcal{O}^{u}\right)$ is dual to $\mathcal{O}_{u}$ in $K^{T}(X)$. More precisely, we have $\chi_{X}\left(\theta_{0}\left(\mathcal{O}^{u}\right) \cdot \mathcal{O}_{v}\right)=$ $\delta_{u, v}$ for $u, v \in W^{P}$.

Proof. We have $\mathcal{O}^{u} \cdot \mathcal{O}_{v}=\left[\mathcal{O}_{X^{u} \cap X_{v}}\right]$ in $K^{T}(X)$. Since $X^{u} \cap X_{v}$ is empty for $u \nless v$ and is rational with rational singularities for $u \leqslant v$ [Ric92, Bri02], we obtain

$$
\chi_{X}\left(\mathcal{O}^{u} \cdot \mathcal{O}_{v}\right)= \begin{cases}1 & \text { if } u \leqslant v, \\ 0 & \text { if } u \nless v .\end{cases}
$$

It follows that $\chi_{X}\left(\theta_{0}\left(\mathcal{O}^{u}\right), \mathcal{O}_{v}\right)$ is the sum of the signs $(-1)^{\ell(w / u)}$ over the set $S$ of all $w \in W^{P}$ for which $u \leqslant w \leqslant v$ and $w / u$ is a rook strip. This sum is equal to 1 if $u=v$, and it is empty if $u \nless v$. Assume that $u<v$, and let $\alpha$ be any minimal box of the skew shape $I(v) \backslash I(u)$. Then the map $w \mapsto w s_{\alpha}$ is an involution of $S$. Since $\ell\left(w s_{\alpha}\right)=\ell(w) \pm 1$ for all $w \in S$, we have $(-1)^{\ell(w / u)}+(-1)^{\ell\left(w s_{\alpha} / u\right)}=0$. This implies that $\chi_{X}\left(\theta_{0}\left(\mathcal{O}^{u}\right), \mathcal{O}_{v}\right)=0$, as required.

For elements $u, w \in W^{P}$ such that $u \leqslant w$ and $w / u$ is a short rook strip, we define an element $\delta(w / u)$ of the root lattice by

$$
\delta(w / u)=\sum_{\alpha \in I(w) \backslash I(u)} \delta(\alpha) .
$$

Equivalently, $\delta(w / u)$ is the sum of the (short) simple roots $\beta \in \Delta$ for which the reflection $s_{\beta}$ appears in a reduced expression for $w / u$. Define an additional homomorphism of $\Gamma$-modules $\phi: K^{T}(X) \rightarrow K^{T}(X)$ by

$$
\phi\left(\mathcal{O}^{u}\right)=\sum_{w / u \text { short rook strip }}(-1)^{\ell(w / u)}\left[\mathbb{C}_{-\delta(w / u)}\right] \mathcal{O}^{w},
$$

where the sum is over all $w \in W^{P}$ for which $u \leqslant w$ and $w / u$ is a short rook strip. If the root system $\Phi$ is simply laced, then $\phi$ is the identity map. The following result is a special case of Lenart and Postnikov's Chevalley formula for the equivariant $K$-theory of arbitrary flag varieties [LP07].

Theorem 3.6. Let $X=G / P$ be a cominuscule space. In $K^{T}(X)$, for any $u \in W^{P}$, we have

$$
J \cdot \mathcal{O}^{u}=J_{u} \theta_{0}\left(\phi\left(\mathcal{O}^{u}\right)\right) .
$$

Theorem 3.6 can be derived from Lenart and Postnikov's more general result as follows. Let $\alpha_{1}, \alpha_{2}, \ldots, \alpha_{s}$ be any ordering of the roots in $\mathcal{P}_{X}$ that is compatible with the partial order $\leqslant$, and let $\beta_{1}, \beta_{2}, \ldots, \beta_{t}$ be an ordering of the short roots of $\mathcal{P}_{X}$ that is compatible with $\leqslant$. Then one can show that the sequence $\left(\alpha_{1}, \ldots, \alpha_{s}, \beta_{1}, \ldots, \beta_{t}\right)$ is what is called a $\lambda$-chain for the weight $\lambda=\omega_{\gamma}$ in [LP07], after which Theorem 3.6 is equivalent to [LP07, Theorem 13.1]. In Section 4, we give an alternative proof of Theorem 3.6 based on geometric considerations.

Notice that since the cominuscule root $\gamma$ is long and the root system $\Phi$ does not have type $\mathrm{G}_{2}$, we have $\left(\omega_{\gamma}, \alpha^{\vee}\right)=2$ for any short root $\alpha \in \mathcal{P}_{X}$. Lemma 3.4 therefore implies that $J_{w}=$ 


\section{A.S. Buch, P.-E. Chaput, L.C. Mihalcea And N. Perrin}

$J_{u}\left[\mathbb{C}_{-2 \delta(w / u)}\right]$ whenever $w / u$ is a short rook strip. For this reason, we will use the notation $\sqrt{J_{u} J_{w}}=J_{u}\left[\mathbb{C}_{-\delta(w / u)}\right]=\left[\mathbb{C}_{u \cdot \omega_{\gamma}-\omega_{\gamma}-\delta(w / u)}\right]$. The identity of Theorem 3.6 can be rewritten as

$$
J \cdot \mathcal{O}^{u}=\sum_{w / u \text { short rook strip }}(-1)^{\ell(w / u)} \sqrt{J_{u} J_{w}} \theta_{0}\left(\mathcal{O}^{w}\right) .
$$

It therefore follows from Lemma 3.5 that Theorem 3.6 is equivalent to the following geometric identity, which is interesting by itself.

Theorem 3.7. For $u, w \in W^{P}$, we have

$$
\chi_{X}\left(J \cdot \mathcal{O}^{u} \cdot \mathcal{O}_{w}\right)= \begin{cases}(-1)^{\ell(w / u)} \sqrt{J_{u} J_{w}} & \text { if } w / u \text { is a short rook strip } \\ 0 & \text { otherwise }\end{cases}
$$

\subsection{A Chevalley formula for $\mathrm{QK}_{T}(X)$}

To state our Chevalley formula for the quantum $K$-theory ring, we define the homomorphism $\theta_{1}: K^{T}(X) \rightarrow K^{T}(X)$ of $\Gamma$-modules as follows. Let $u \in W^{P}$. If $u \geqslant z_{1}$, then we set

$$
\theta_{1}\left(\mathcal{O}^{u}\right)=\sum_{w}(-1)^{\ell(w / u(-1))} \mathcal{O}^{w},
$$

where the sum is over all $w \in W^{P}$ for which $u(-1) \leqslant w \leqslant 1^{\vee}(-1)=z_{1}^{\vee}$ and $w / u(-1)$ is a rook strip. If $u \ngtr z_{1}$, then we set $\theta_{1}\left(\mathcal{O}^{u}\right)=0$.

Lemma 3.8. We have $\theta_{1}=\psi \theta_{0}$ as $\Gamma$-linear endomorphisms of $K^{T}(X)$.

Proof. For any $u \in W^{P}$, we have

$$
\psi\left(\theta_{0}\left(\mathcal{O}^{u}\right)\right)=\sum_{w / u \text { rook strip }}(-1)^{\ell(w / u)} \mathcal{O}^{w(-1)} .
$$

If $u \geqslant z_{1}$, then we have $w(-1)=w / z_{1}$ and $w(-1) / u(-1)=w / u$ for each element $w$ in the sum. Since the map $w \mapsto w(-1)$ gives a bijection between the terms of (3.1) and the sum defining $\theta_{1}\left(\mathcal{O}^{u}\right)$, the lemma follows in this case. If $u \ngtr z_{1}$, let $\alpha$ be a minimal box in the set $I\left(z_{1}\right) \backslash I(u)$. Then the map $w \mapsto w s_{\alpha}$ defines an involution of the set of elements $w$ appearing in (3.1). Since we have $\ell\left(w s_{\alpha}\right)=\ell(w) \pm 1$ and the identity $w(-1)=\left(w \cup z_{1}\right) / z_{1}$ implies that $w(-1)=\left(w s_{\alpha}\right)(-1)$, we deduce that $\psi\left(\theta_{0}\left(\mathcal{O}^{u}\right)\right)=0$, as required.

Theorem 3.9. Let $X=G / P$ be cominuscule. In $\mathrm{QK}_{T}(X)$, for $u \in W^{P}$, we have

$$
J \star \mathcal{O}^{u}=J_{u} \theta_{0}\left(\phi\left(\mathcal{O}^{u}\right)\right)-J_{u} q \theta_{1}\left(\phi\left(\mathcal{O}^{u}\right)\right) .
$$

Proof. Since all curves of positive degree in $X$ meet the Schubert divisor $X^{s_{\gamma}}$, we have $\Gamma_{d}\left(X_{u}, X^{s_{\gamma}}\right)=\Gamma_{d}\left(X_{u}\right)=\Gamma_{d}\left(X_{u}, X\right)$ for all $d \geqslant 1$. Theorem 3.3 therefore implies that

$$
I_{d}\left(\mathcal{O}_{u}, J, \mathcal{O}_{w}^{\vee}\right)=I_{d}\left(\mathcal{O}_{u}, 1, \mathcal{O}_{w}^{\vee}\right)-I_{d}\left(\mathcal{O}_{u}, \mathcal{O}^{s_{\gamma}}, \mathcal{O}_{w}^{\vee}\right)=0
$$

for all $u, w \in W^{P}$ and $d \geqslant 1$. By linearity, we obtain $I_{d}\left(\sigma, J, \mathcal{O}_{w}^{\vee}\right)=0$ for all $\sigma \in K^{T}(X)$ and $d \geqslant 1$, which is equivalent to the identity $\sigma \odot J=\sigma \cdot J$. We therefore obtain

$$
\begin{aligned}
J \star \mathcal{O}^{u} & =(1-q \psi)\left(J \odot \mathcal{O}^{u}\right)=(1-q \psi)\left(J \cdot \mathcal{O}^{u}\right) \\
& =(1-q \psi)\left(J_{u} \theta_{0}\left(\phi\left(\mathcal{O}^{u}\right)\right)\right)=J_{u} \theta_{0}\left(\phi\left(\mathcal{O}^{u}\right)\right)-J_{u} q \psi\left(\theta_{0}\left(\phi\left(\mathcal{O}^{u}\right)\right)\right),
\end{aligned}
$$

so the result follows from Lemma 3.8. 


\section{A Chevalley formula for equivariant quantum $K$-Theory}

\subsection{The structure constants of the Chevalley formula}

Recall that a box $\alpha$ of a skew shape $I(w) \backslash I(u)$ is incomparable if it is both a minimal and maximal box within this skew shape. Theorem 3.9 can be restated as follows.

Corollary 3.10. Let $X=G / P$ be cominuscule. For $u \in W^{P}$, we have

$$
\mathcal{O}^{s_{\gamma}} \star \mathcal{O}^{u}=\sum_{w \in W^{P}} N_{s_{\gamma}, u}^{w, 0} \mathcal{O}^{w}+\sum_{w \in W^{P}} N_{s_{\gamma}, u}^{w, 1} q \mathcal{O}^{w}
$$

where the constants are determined by $N_{s_{\gamma}, u}^{u, 0}=1-J_{u}$ and the following rules.

For $u \neq w$, the constant $N_{s_{\gamma}, u}^{w, 0}$ is non-zero if and only if $u<w$ and all non-maximal boxes of the skew shape $I(w) \backslash I(u)$ are short minimal boxes. In this case, we have

$$
N_{s_{\gamma}, u}^{w, 0}=(-1)^{\ell(w / u)-1} J_{u}\left(\prod_{\alpha}\left[\mathbb{C}_{-\delta(\alpha)}\right]\right)\left(\prod_{\alpha^{\prime}}\left(1+\left[\mathbb{C}_{-\delta\left(\alpha^{\prime}\right)}\right]\right)\right),
$$

where the first product is over all non-maximal boxes $\alpha$ of $I(w) \backslash I(u)$ and the second product is over all short incomparable boxes $\alpha^{\prime}$ of $I(w) \backslash I(u)$.

The constant $N_{s_{\gamma}, u}^{w, 1}$ is non-zero if and only if $u(-1) \leqslant w \leqslant z_{1}^{\vee}$, all non-maximal boxes of the skew shape $I(w(1)) \backslash I(u)$ are short minimal boxes, and all roots of $I\left(z_{1}\right) \backslash I(u)$ are short minimal boxes of $I(w(1)) \backslash I(u)$. In this case, we have

$$
N_{s_{\gamma}, u}^{w, 1}=(-1)^{\ell(w(1) / u)} J_{u}\left(\prod_{\alpha}\left[\mathbb{C}_{-\delta(\alpha)}\right]\right)\left(\prod_{\alpha^{\prime}}\left(1+\left[\mathbb{C}_{-\delta\left(\alpha^{\prime}\right)}\right]\right)\right),
$$

where the first product is over all boxes $\alpha$ of $I(w(1)) \backslash I(u)$ that are either non-maximal or belong to $I\left(z_{1}\right)$ and the second product is over all short incomparable boxes $\alpha^{\prime}$ of $I(w(1)) \backslash I(u)$ for which $\alpha^{\prime} \notin I\left(z_{1}\right)$.

Proof. Theorem 3.9 implies that the coefficient of $q \mathcal{O}^{w}$ in $J \star \mathcal{O}^{u}$ is given by

$$
-N_{s_{\gamma}, u}^{w, 1}=(-1)^{\ell(w)+\ell\left(z_{1}\right)+1-\ell(u)} J_{u} \sum_{v}\left[\mathbb{C}_{-\delta(v / u)}\right],
$$

where the sum is over all $v \in W^{P}$ for which $\mathcal{O}^{v}$ appears in $\phi\left(\mathcal{O}^{u}\right)$ and $\mathcal{O}^{w}$ appears in $\theta_{1}\left(\mathcal{O}^{v}\right)$. The first condition says that $u \leqslant v$ and $v / u$ is a short rook strip, and by Lemma 3.1, the second condition holds if and only if $w \leqslant z_{1}^{\vee}, z_{1} \leqslant v \leqslant w(1)$, and $w(1) / v$ is an arbitrary rook strip. Such elements $v$ exist if and only if the conditions for $N_{s_{\gamma}, u}^{w, 1} \neq 0$ in the corollary are satisfied, and in this case, $v$ appears in the sum if and only if $I(v)$ is the union of $I(u)$, the set of all roots $\alpha$ in the first product of (3.3), and an arbitrary subset of the roots $\alpha^{\prime}$ in the second product. The identity (3.3) now follows because the last two products in this identity expand to the sum in equation (3.4). The proof of (3.2) is similar and left to the reader.

Remark 3.11. Assume that the root system $R$ is simply laced. Then there are no short boxes, and the statement of Corollary 3.10 simplifies considerably. Any coefficient $N_{s_{\gamma}, u}^{w, 0}$ with $w \neq u$ is non-zero if and only if $u<w$ and $w / u$ is a rook strip, in which case

$$
N_{s_{\gamma}, u}^{w, 0}=(-1)^{\ell(w / u)-1} J_{u} .
$$

Similarly, $N_{s_{\gamma}, u}^{w, 1}$ is non-zero if and only if $u(-1) \leqslant w \leqslant z_{1}^{\vee}, z_{1} \leqslant u$, and $w(1) / u$ is a rook strip. In this case, we have

$$
N_{s_{\gamma}, u}^{w, 1}=(-1)^{\ell(w(1) / u)} J_{u}
$$




\section{A.S. Buch, P.-E. Chaput, L.C. Mihalcea And N. Perrin}

We proceed to give two examples of the Chevalley formula in types A and C. More examples can be obtained using the Equivariant Schubert Calculator available at http://sites.math. rutgers.edu/ asbuch/equivcalc/.

EXAmple 3.12. Let $X=\operatorname{Gr}(3,7)=\left\{V \subset \mathbb{C}^{7} \mid \operatorname{dim}(V)=3\right\}$ be the Grassmann variety of three-planes in $\mathbb{C}^{7}$. This is a homogeneous space for the group $G=\mathrm{SL}\left(\mathbb{C}^{7}\right)$ of Lie type $\mathrm{A}_{6}$. Let $T \subset G$ be the maximal torus of diagonal matrices, and let $B \subset G$ be the Borel subgroup of uppertriangular matrices. For $1 \leqslant i \leqslant 7$, we let $\varepsilon_{i}: T \rightarrow \mathbb{C}^{*}$ be the character that sends a diagonal matrix to its $i$ th entry. Then the set of simple roots of $G$ is $\Delta=\left\{\varepsilon_{i}-\varepsilon_{i+1} \mid 1 \leqslant i \leqslant 6\right\}$, and all these simple roots are cominuscule. We can identify the homogeneous space $G / P$ corresponding to $\gamma=\varepsilon_{3}-\varepsilon_{4}$ with the Grassmannian $X$ by the map $g . P \mapsto g . E_{3}$, where $E_{3}=\operatorname{Span}\left\{e_{1}, e_{2}, e_{3}\right\}$ is the span of the first three standard basis vectors in $\mathbb{C}^{7}$. The partially ordered set $\mathcal{P}_{X}$ consists of the boxes of the following $3 \times 4$ rectangle:

\begin{tabular}{|c|l|l|l|}
\hline$\gamma$ & $\varepsilon_{3}-\varepsilon_{5}$ & $\varepsilon_{3}-\varepsilon_{6}$ & $\varepsilon_{3}-\varepsilon_{7}$ \\
\hline$\varepsilon_{2}-\varepsilon_{4}$ & $\varepsilon_{2}-\varepsilon_{5}$ & $\varepsilon_{2}-\varepsilon_{6}$ & $\varepsilon_{2}-\varepsilon_{7}$ \\
\hline$\varepsilon_{1}-\varepsilon_{4}$ & $\varepsilon_{1}-\varepsilon_{5}$ & $\varepsilon_{1}-\varepsilon_{6}$ & $\varepsilon_{1}-\varepsilon_{7}$ \\
\hline
\end{tabular}

The partial order is given by $\varepsilon_{i}-\varepsilon_{j} \leqslant \varepsilon_{k}-\varepsilon_{l}$ if and only if $k \leqslant i$ and $j \leqslant l$ or, equivalently, the box $\varepsilon_{i}-\varepsilon_{j}$ is north-west of $\varepsilon_{k}-\varepsilon_{l}$. The order ideals of $\mathcal{P}_{X}$ therefore correspond to Young diagrams $\lambda$ contained in the upper-left corner of the rectangle. Notice that $\delta\left(\varepsilon_{i}-\varepsilon_{j}\right)=\varepsilon_{i+j-4}-\varepsilon_{i+j-3}$. Since we have $I\left(z_{1}\right)=\bigoplus^{\Pi}$, the map $w \mapsto w(-1)$ corresponds to the map of Young diagrams that removes the first row and the first column and moves the remaining boxes one step north-west. Similarly, the map $w \mapsto w(1)$ corresponds to the map of Young diagrams that moves all boxes one step south-east, discards any boxes that leave the $3 \times 4$ rectangle, and adds the boxes of $I\left(z_{1}\right)$ to the result. If $w \leqslant z_{1}^{\vee}$, then no boxes need to be discarded; equivalently, we have $w(1)(-1)=w$.

Consider the diagram $\mu=\bigoplus$, and let $w_{\mu} \in W^{P}$ be the corresponding Weyl group element. Using Lemma 3.4, we obtain $J_{w_{\mu}}=\left[\mathbb{C}_{\varepsilon_{7}+\varepsilon_{5}-\varepsilon_{3}-\varepsilon_{1}}\right]$. We will abuse notation and denote the Schubert class $\mathcal{O}^{w_{\lambda}}$ simply by $\lambda$. Since $\phi\left(\mathcal{O}^{w_{\mu}}\right)=\mathcal{O}^{w_{\mu}}$ and $I\left(w_{\mu}(-1)\right)=\square$, it follows from Theorem 3.9 that

$$
J \star \boxplus=J_{w_{\mu}}(\boxplus-\boxplus \square-\boxplus+\bigoplus-q \square+q \square+q \boxminus-q \boxminus) .
$$

Equivalently, the expansion of the product $\mathcal{O}^{s_{\gamma}} \star \mathcal{O}^{w_{\mu}}=\mathcal{O}^{w_{\mu}}-J \star \mathcal{O}^{w_{\mu}}$ can be obtained using Remark 3.11.

EXAMPLE 3.13 (Lagrangian Grassmannian). Let $E=\mathbb{C}^{2 n}$, let $\left\{e_{1}, e_{2}, \ldots, e_{2 n}\right\}$ be the standard basis, and define a symplectic form on $E$ by $\left(e_{i}, e_{j}\right)=-\left(e_{j}, e_{i}\right)=\delta_{i+j, 2 n+1}$ for $1 \leqslant i \leqslant j \leqslant 2 n$. The Lagrangian Grassmannian of maximal isotropic subspaces of $E$ is the variety

$$
X=\mathrm{LG}(n, E)=\{V \subset E \mid \operatorname{dim}(V)=n \text { and }(V, V)=0\} .
$$

This is a homogeneous space for the symplectic group $G=\operatorname{Sp}(E)=\{g \in \operatorname{GL}(E) \mid(g . x, g . y)=$ $(x, y) \forall x, y \in E\}$ of Lie type $\mathrm{C}_{n}$. Let $T \subset G$ be the maximal torus of diagonal matrices, and let $B \subset G$ be the Borel subgroup of upper-triangular matrices. For $1 \leqslant i \leqslant 2 n$, we let $\varepsilon_{i}: T \rightarrow \mathbb{C}^{*}$ be the character that sends a diagonal matrix to its $i$ th entry. Since all elements of $T$ have the form $\operatorname{diag}\left(t_{1}, \ldots, t_{n}, t_{n}^{-1}, \ldots, t_{1}^{-1}\right)$, we have $\varepsilon_{2 n+1-i}=-\varepsilon_{i}$ for each $i$. The set of simple roots 


\section{A Chevalley formula for equivariant quantum $K$-Theory}

of $G$ is $\Delta=\left\{\varepsilon_{1}-\varepsilon_{2}, \varepsilon_{2}-\varepsilon_{3}, \ldots, \varepsilon_{n-1}-\varepsilon_{n}, 2 \varepsilon_{n}\right\}$. This set contains a single cominuscule simple root $\gamma=2 \varepsilon_{n}$. The corresponding homogeneous space $G / P$ can be identified with $X$ by the map g.P $\mapsto g . E_{n}$, where $E_{n}=\operatorname{Span}\left\{e_{1}, \ldots, e_{n}\right\}$. The partially ordered set $\mathcal{P}_{X}$ consists of the boxes in the staircase diagram

\begin{tabular}{|c|c|c|c|c|}
\hline$\gamma$ & $\cdots$ & $\varepsilon_{3}+\varepsilon_{n}$ & $\varepsilon_{2}+\varepsilon_{n}$ & $\varepsilon_{1}+\varepsilon_{n}$ \\
\hline & $\ddots$ & $\vdots$ & $\vdots$ & $\vdots$ \\
\hline & $2 \varepsilon_{3}$ & $\varepsilon_{2}+\varepsilon_{3}$ & $\varepsilon_{1}+\varepsilon_{3}$ \\
\hline & $2 \varepsilon_{2}$ & $\varepsilon_{1}+\varepsilon_{2}$ \\
\cline { 2 - 4 } & \multicolumn{1}{|c|}{} & $2 \varepsilon_{1}$ \\
\hline
\end{tabular}

where boxes increase from north-west to south-east as in Example 3.12. The long roots of $\mathcal{P}_{X}$ are the $n$ diagonal boxes. Each element $u \in W^{P}$ corresponds to a shifted Young diagram $I(u)$ of boxes in the upper-left corner of the staircase diagram. Since $I\left(z_{1}\right)$ is the top row of boxes in $\mathcal{P}_{X}$, the map $w \mapsto w(-1)$ corresponds to the map of shifted Young diagrams that removes the top row and moves the remaining boxes one step north-west. The map $w \mapsto w(1)$ corresponds to the map of shifted Young diagrams that moves all boxes one step south-east, discards the boxes that leave the staircase diagram $\mathcal{P}_{X}$, and adds the boxes of $I\left(z_{1}\right)$ to the result. If $I(w)$ does not contain boxes in the right-most column of $\mathcal{P}_{X}$, then no boxes are discarded.

To illustrate the Chevalley formula, we consider the case $n=5$ and $\mu=\square$. If we denote each Schubert class $\mathcal{O}^{w_{\lambda}}$ by $\lambda$ as above, then we have

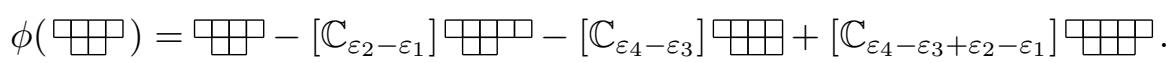

Lemma 3.4 gives $J_{w_{\mu}}=\left[\mathbb{C}_{-2\left(\varepsilon_{2}+\varepsilon_{4}\right)}\right]$, and Theorem 3.9 shows that the quantum terms of the product $J \star \mathcal{O}^{w_{\mu}}$ are given by $-J_{w_{\mu}} q \theta_{1}\left(\phi\left(\mathcal{O}^{w_{\mu}}\right)\right)$, which expands to

$$
q\left[\mathbb{C}_{-\varepsilon_{1}-\varepsilon_{2}-2 \varepsilon_{4}}\right](\square-\square-\square+\square)-q\left[\mathbb{C}_{-\varepsilon_{1}-\varepsilon_{2}-\varepsilon_{3}-\varepsilon_{4}}\right](\square-\square-\square+\square) .
$$

Alternatively, we can use Corollary 3.10 to compute the coefficients $N_{s_{\gamma}, w_{\mu}}^{w_{\nu}, 1}$ in the expansion of $\mathcal{O}^{s_{\gamma}} \star \mathcal{O}^{w_{\mu}}$. For example, for $\nu=\square$, we obtain $I\left(w_{\nu}(1)\right)=\Psi$, and noting that $I\left(z_{1}\right) \backslash \mu$ consists of the single short box $\varepsilon_{1}+\varepsilon_{5}$, we obtain

$$
N_{s_{\gamma}, w_{\mu}}^{w_{\nu}, 1}=(-1)^{3} J_{u}\left[\mathbb{C}_{\varepsilon_{2}-\varepsilon_{1}}\right]\left(1+\left[\mathbb{C}_{\varepsilon_{4}-\varepsilon_{3}}\right]\right) .
$$

\section{A geometric proof of the Chevalley formula}

\subsection{Minuscule varieties}

This section contains a geometric proof of Theorem 3.6. As in the previous section, we assume that $X=G / P$ is a cominuscule variety, and $\gamma \in \Delta$ is the corresponding cominuscule simple root. While our proof is very simple when the root system $\Phi$ is simply laced, we need to resort to specialized arguments in the non-simply laced cases.

We may assume without loss of generality that $G$ is simply connected, so that any integral weight of the root system $\Phi$ is represented by a character of $T$. The fundamental weight $\omega_{\gamma}$ extends to a character $\omega_{\gamma}: P \rightarrow \mathbb{C}^{*}$. Let $L=G \times{ }^{P} \mathbb{C}_{-\omega_{\gamma}}$ be the associated line bundle on $X$. The 


\section{A.S. Buch, P.-E. Chaput, L.C. Mihalcea and N. Perrin}

total space of this bundle consists of all pairs $[g, z]$ with $g \in G$ and $z \in \mathbb{C}$, subject to the relation $\left[g p, \omega_{\gamma}(p) z\right]=[g, z]$ for all $p \in P$. We regard $L$ as a $G$-equivariant line bundle with action defined by $g^{\prime} \cdot[g, z]=\left[g^{\prime} g, z\right]$. In particular, $G$ acts on the vector space of global sections $H^{0}(X, L)$. According to the Borel-Weil theorem, $H^{0}(X, L)^{*}$ is an irreducible representation of $G$ with highest weight $\omega_{\gamma}$. It follows that there exists a $B^{-}$-stable section $\sigma \in H^{0}(X, L)$ of weight $-\omega_{\gamma}$. Given any element $u \in W$, we will misuse notation and write $u . \sigma=\dot{u} \cdot \sigma$, where $\dot{u} \in N_{G}(T)$ is a representative of $u$. The section $u . \sigma \in H^{0}(X, L)$ is well defined only up to a scalar.

Proposition 4.1. Let $u \in W^{P}$. The support of $Z(u . \sigma) \cap X^{u}$ is the boundary $\partial X^{u}$. If all minimal boxes of $\mathcal{P}_{X} \backslash I(u)$ are long roots, then $Z(u . \sigma) \cap X^{u}=\partial X^{u}$ as (reduced) subschemes of $X^{u}$. In particular, if $X$ is a minuscule variety, then the boundary $\partial X^{u}$ is a Cartier divisor in $X^{u}$.

Proof. Since $\sigma$ is a $B^{-}$-stable section of $L$, it follows that the zero section $Z(\sigma)$ is a $B^{-}$-stable divisor, so we have $Z(\sigma)=X^{s_{\gamma}}$ as a set. It follows that $1 . P \notin Z(\sigma)$, and hence $u . P \notin Z(u . \sigma)$, so Lemma 2.1 implies that $Z(u . \sigma) \cap X^{u} \subset \partial X^{u}$. Let $\tau \in H^{0}\left(X^{u},\left.L\right|_{X^{u}}\right)$ denote the section obtained by restricting $u . \sigma$ to $X^{u}$. The cycle $[Z(\tau)]$ is a sum of prime divisors of $X^{u}$ contained in the boundary $\partial X^{u}$. These prime divisors are exactly the Schubert varieties $X^{w}$ for which $w$ covers $u$ in the Bruhat order on $W^{P}$; that is, $u \leqslant w$ and $\ell(w)=\ell(u)+1$. Furthermore, the classes $\left[X^{w}\right]$ of these prime divisors are linearly independent in $H_{*}\left(X^{u} ; \mathbb{Z}\right)$ as they map to linearly independent classes in $H_{*}(X ; \mathbb{Z})$. For each $w \in W^{P}$ that covers $u$, we have $I(w) \backslash I(u)=\{\alpha\}$ for some root $\alpha \in \mathcal{P}_{X}$ such that $w=u s_{\alpha}$, and it follows from the classical Chevalley formula [Che94] that the coefficient of $\left[X^{w}\right]$ in $[Z(\tau)]$ is equal to $\left(\omega_{\gamma}, \alpha^{\vee}\right)$. Since the cominuscule root $\gamma$ is long, and since the coefficient of $\gamma$ in $\alpha$ is 1 , it follows that $\left(\omega_{\gamma}, \alpha^{\vee}\right)$ is equal to 1 if $\alpha$ is long and equal to 2 if $\alpha$ is short. In particular, $\tau$ vanishes along each Schubert divisor contained in $\partial X^{u}$, so we have $Z(u . \sigma) \cap X^{u}=Z(\tau)=\partial X^{u}$ as sets. If all minimal boxes of $\mathcal{P}_{X} \backslash I(u)$ are long roots, then $\tau$ vanishes to the first order along each prime divisor $X^{w} \subset X^{u}$. In other words, the ideal of $Z(\tau)$ agrees with the maximal ideal in each discrete valuation ring $\mathcal{O}_{X^{w}, X^{u}}$. By using that $X^{u}$ is normal and that any normal ring is the intersection of its localizations at prime ideals of codimension 1 , we deduce that $Z(\tau)$ is a reduced subscheme of $X^{u}$. This completes the proof.

It follows from Proposition 4.1 that $X^{s_{\gamma}}=Z(\sigma)$ as a subscheme of $X$. Since $\sigma$ has weight $-\omega_{\gamma}$ in $H^{0}(X, L)$, we may consider $\sigma$ as a morphism $\sigma: X \times \mathbb{C}_{-\omega_{\gamma}} \rightarrow L$ of $T$-equivariant vector bundles on $X$. We obtain a $T$-equivariant exact sequence

$$
0 \rightarrow L^{\vee} \otimes \mathbb{C}_{-\omega_{\gamma}} \rightarrow \mathcal{O}_{X} \rightarrow \mathcal{O}_{X^{s}} \rightarrow 0
$$

which reveals the identity $J=1-\mathcal{O}^{s_{\gamma}}=\left[L^{\vee} \otimes \mathbb{C}_{-\omega_{\gamma}}\right]$ in $K^{T}(X)$.

Proof of Lemma 3.4. The first identity of the lemma follows since $\left.L\right|_{u . P} \cong \mathbb{C}_{-u . \omega_{\gamma}}$ as a representation of $T$. The second identity is clear if $u=1$. If $u \neq 1$, then choose $u^{\prime} \in W^{P}$ such that $u^{\prime} \leqslant u$ and $\ell\left(u^{\prime}\right)=\ell(u)-1$. Then $I(u)=I\left(u^{\prime}\right) \cup\{\alpha\}$ for some root $\alpha \in \mathcal{P}_{X}$ such that $u=u^{\prime} s_{\alpha}$, and we have $\delta(\alpha)=u^{\prime} . \alpha$. We obtain

$$
\omega_{\gamma}-u \cdot \omega_{\gamma}=\omega_{\gamma}-u^{\prime} \cdot\left(\omega_{\gamma}-\left(\omega_{\gamma}, \alpha^{\vee}\right) \alpha\right)=\left(\omega_{\gamma}-u^{\prime} \cdot \omega_{\gamma}\right)+\left(\omega_{\gamma}, \alpha^{\vee}\right) \delta(\alpha),
$$

so the required identity follows by induction on $\ell(u)$.

The following result implies Theorem 3.6 for all minuscule varieties.

Proposition 4.2. Theorem 3.7 is true whenever all minimal boxes of $\mathcal{P}_{X} \backslash I(u)$ are long roots. 


\section{A Chevalley formula for equivariant quantum $K$-Theory}

Proof. In the situation of the proposition, Theorem 3.7 states that $J_{u}^{-1} J \mathcal{O}^{u}$ is dual to $\mathcal{O}_{u}$. Proposition 4.1 implies that $\left.I_{\partial X^{u}} \cong\left(L^{\vee} \otimes \mathbb{C}_{-u . \omega_{\gamma}}\right)\right|_{X^{u}}$, so $J_{u}^{-1} J \mathcal{O}^{u}=\left[L^{\vee} \otimes \mathbb{C}_{-u . \omega_{\gamma}}\right] \mathcal{O}^{u}=\left[I_{\partial X^{u}}\right]$ is dual to $\mathcal{O}_{u}$ by Brion's identity (2.1).

\subsection{Lagrangian Grassmannians}

We next prove Theorem 3.7 for Lagrangian Grassmannians $X=\mathrm{LG}(n, E)$, using the notation introduced in Example 3.13.

Let $\mathbb{P} E$ be the projective space of lines through the origin in $E$, set $\operatorname{SF}(1, n ; E)=\{(L, V) \in$ $\mathbb{P} E \times X \mid L \subset V\}$, and let $\pi: \mathrm{SF}(1, n ; E) \rightarrow X$ and $\varphi: \mathrm{SF}(1, n ; E) \rightarrow \mathbb{P} E$ be the projections. Both of these projections are $T$-equivariant maps. Set $E^{n}=\operatorname{Span}\left\{e_{n+1}, e_{n+2}, \ldots, e_{2 n}\right\}$, and notice that the opposite Schubert divisor in $X$ is defined by $X^{s_{\gamma}}=\left\{V \in X \mid V \cap E^{n} \neq 0\right\}=\pi\left(\varphi^{-1}\left(\mathbb{P} E^{n}\right)\right)$. Since $\pi$ maps $\varphi^{-1}\left(\mathbb{P} E^{n}\right)$ birationally onto $X^{s_{\gamma}}$, we obtain $\mathcal{O}^{s_{\gamma}}=\pi_{*} \varphi^{*}\left[\mathcal{O}_{\mathbb{P} E^{n}}\right]$ in $K^{T}(X)$.

Let $u, w \in W^{P}$ be elements satisfying $u \leqslant w$. Then we have $\mathcal{O}^{u} \cdot \mathcal{O}_{w}=\left[\mathcal{O}_{X^{u} \cap X_{w}}\right]$ and $\chi_{X}\left(\mathcal{O}^{u} \cdot \mathcal{O}_{w}\right)=1$. Set $Z_{w}^{u}=\varphi\left(\pi^{-1}\left(X^{u} \cap X_{w}\right)\right) \subset \mathbb{P} E$. It follows from [BR12, Lemma 5.1] or $\left[\right.$ KLS14, Theorem 4.5] that $\varphi_{*} \pi^{*}\left(\mathcal{O}^{u} \cdot \mathcal{O}_{w}\right)=\left[\mathcal{O}_{Z_{w}^{u}}\right]$ in $K^{T}(\mathbb{P} E)$. By the projection formula, we therefore obtain

$$
\chi_{X}\left(J \cdot \mathcal{O}^{u} \cdot \mathcal{O}_{w}\right)=\chi_{X}\left(\mathcal{O}^{u} \cdot \mathcal{O}_{w}\right)-\chi_{X}\left(\mathcal{O}^{s_{\gamma}} \cdot \mathcal{O}^{u} \cdot \mathcal{O}_{w}\right)=1-\chi_{\mathbb{P E}}\left(\left[\mathcal{O}_{\mathbb{P} E^{n}}\right] \cdot\left[\mathcal{O}_{Z_{w}^{u}}\right]\right) .
$$

It is known from [BR12, Lemma 5.2] that $Z_{w}^{u}$ is a complete intersection in $\mathbb{P} E$ defined by linear and quadratic equations. We need the following explicit description of these equations, which is a consequence of the proof given in [BR12]. Notice that the south-east boundary of the shape $I(u)$ is a path of $n$ line segments from the upper-right corner of the staircase diagram $\mathcal{P}_{X}$ to its diagonal (see Example 4.3). The $k$ th step of this path will be called the $k$ th boundary segment of $u$. Let $\mathbb{C}\left[x_{1}, \ldots, x_{2 n}\right]$ be the projective coordinate ring of $\mathbb{P} E$, with $x_{i}$ dual to the basis element $e_{i} \in E$. The linear equations of $Z_{w}^{u}$ correspond to the boundary segments that $u$ and $w$ have in common. Assume that the $k$ th boundary segment of $u$ is also a boundary segment of $w$. If this boundary segment is horizontal, then it contributes the linear equation $x_{2 n+1-k}=0$, and if it is vertical, then it contributes the equation $x_{k}=0$. Two boxes in the staircase diagram are connected if they share a side. The quadratic equations of $Z_{w}^{u}$ correspond to connected components of the skew shape $I(w) \backslash I(u)$ which do not contain any (long) diagonal boxes of $\mathcal{P}_{X}$. These components will be called short components of $w / u$. Assume that the north-west side of a short component consists of the boundary segments of $u$ numbered from $i$ to $j$. Then the component contributes the quadratic equation $f_{i j}=x_{i} x_{2 n+1-i}+x_{i+1} x_{2 n-i}+\cdots+x_{j} x_{2 n+1-j}=0$.

ExAmple 4.3. Let $X=\mathrm{LG}(7,14)$ be the Lagrangian Grassmannian of type $\mathrm{C}_{7}$, and let $u \leqslant w$ be the elements of $W^{P}$ corresponding to the marked boundaries:

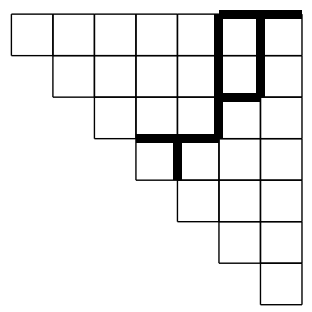

Then $Z_{w}^{u} \subset \mathbb{P}^{13}$ is defined by $x_{5}=x_{9}=x_{14}=x_{2} x_{13}+x_{3} x_{12}+x_{4} x_{11}=0$.

Let $\mathcal{O}_{\mathbb{P} E}(-1) \subset \mathbb{P} E \times E$ denote the tautological subbundle, with the equivariant structure defined by the action of $G$ on $E$, and set $h=\left[\mathcal{O}_{\mathbb{P} E}(-1)\right] \in K^{T}(\mathbb{P} E)$. Since $T$ acts on $x_{i}$ with 


\section{A.S. Buch, P.-E. Chaput, L.C. Mihalcea and N. Perrin}

weight $-\varepsilon_{i}$ and on $f_{i j}$ with weight $\varepsilon_{i}+\varepsilon_{2 n+1-i}=0$, we have $\left[\mathcal{O}_{Z\left(x_{i}\right)}\right]=1-\left[\mathbb{C}_{-\varepsilon_{i}}\right] h$ and $\left[\mathcal{O}_{Z\left(f_{i j}\right)}\right]=$ $1-h^{2}$ in $K^{T}(\mathbb{P} E)$. Let $l$ be the number of boundary segments shared by $u$ and $w$, let $\mu_{1}, \mu_{2}, \ldots, \mu_{l}$ be the weights of the corresponding linear equations, and let $q$ be the number of short components of $w / u$. We then have

$$
\left[\mathcal{O}_{\mathbb{P} E^{n}}\right] \cdot\left[\mathcal{O}_{Z_{w}^{u}}\right]=\left(\prod_{j=1}^{n}\left(1-\left[\mathbb{C}_{-\varepsilon_{j}}\right] h\right)\right)\left(\prod_{i=1}^{l}\left(1-\left[\mathbb{C}_{\mu_{i}}\right] h\right)\right)\left(1-h^{2}\right)^{q}=1+\sum_{k=1}^{n+l+2 q} a_{k} h^{k}
$$

for some classes $a_{k} \in \Gamma$. The leading coefficient is $a_{n+l+2 q}=(-1)^{n+l+q}\left[\mathbb{C}_{\nu(u, w)}\right]$, where $\nu(u, w)=$ $\sum_{i=1}^{l} \mu_{i}-\sum_{j=1}^{n} \varepsilon_{j}$.

Lemma 4.4. Let $F$ be any representation of $T$ of dimension $d$, and consider the sheaf Euler characteristic map $\chi_{\mathbb{P} F}: K^{T}(\mathbb{P} F) \rightarrow \Gamma$. Then we have

$$
\chi_{\mathbb{P F}}\left(\left[\mathcal{O}_{\mathbb{P} F}(-k)\right]\right)= \begin{cases}1 & \text { if } k=0, \\ 0 & \text { if } 0<k<d, \\ (-1)^{d-1}\left[\bigwedge^{d} F\right] & \text { if } k=d .\end{cases}
$$

Proof. This follows from Serre duality [Har77, III.7.7] because the canonical sheaf on $\mathbb{P} F$ is given by $\omega_{\mathbb{P} F}=\left(\bigwedge^{d} F^{*}\right) \otimes \mathcal{O}_{\mathbb{P} F}(-d)$ as a $T$-equivariant vector bundle.

Since each short component of $w / u$ occupies at least two of the boundary segments of $u$, we have $l+2 q \leqslant n$, with equality if and only if $w / u$ is a short rook strip, and in this case we have $\ell(w / u) \equiv n+l+q(\bmod 2)$. Since $\left[\bigwedge^{2 n} E\right]=1 \in \Gamma$, we obtain

$$
\chi_{X}\left(J \cdot \mathcal{O}^{u} \cdot \mathcal{O}_{w}\right)= \begin{cases}(-1)^{\ell(w / u)}\left[\mathbb{C}_{\nu(u, w)}\right] & \text { if } w / u \text { is a short rook strip }, \\ 0 & \text { otherwise } .\end{cases}
$$

As special cases, we have $J_{u}=\chi_{X}\left(J \cdot \mathcal{O}^{u} \cdot \mathcal{O}_{u}\right)=\left[\mathbb{C}_{\nu(u, u)}\right]$ and $J_{w}=\left[\mathbb{C}_{\nu(w, w)}\right]$. To finish the proof of Theorem 3.7, it is therefore enough to show that $2 \nu(u, w)=\nu(u, u)+\nu(w, w)$ whenever $w / u$ is a short rook strip. To see this, let $\varepsilon_{i}+\varepsilon_{j} \in I(w) \backslash I(u)$, and set $k=n+i-j$. Then the $k$ th boundary segment of $u$ is horizontal, while the $k$ th boundary segment of $w$ is vertical, so $Z_{u}^{u}$ satisfies the equation $x_{2 n+1-k}=0$ while $Z_{w}^{w}$ satisfies the equation $x_{k}=0$. It follows that the weights $-\varepsilon_{2 n+1-k}$ and $-\varepsilon_{k}$ of these equations cancel out in the sum $\nu(u, u)+\nu(w, w)$, and the same happens for the weights corresponding to the $(k+1)$ st boundary segments. This shows that $2 \nu(u, w)=\nu(u, u)+\nu(w, w)$ and completes the proof.

\subsection{Odd quadric hypersurfaces}

We finally prove Theorem 3.6 for odd quadric hypersurfaces, which is the last case needed to complete the proof of the Chevalley formula. Set $E=\mathbb{C}^{2 n+1}$, with basis $\left\{e_{1}, e_{2}, \ldots, e_{2 n+1}\right\}$, and define an orthogonal form on $E$ by $\left(e_{i}, e_{j}\right)=\delta_{i+j, 2 n+2}$. The corresponding symmetry group $G=\mathrm{SO}(E)$ has Lie type $\mathrm{B}_{n}$. Let $T \subset G$ be the maximal torus of diagonal matrices, and let $B \subset G$ be the Borel subgroup of upper-triangular matrices. For $1 \leqslant i \leqslant 2 n+1$, we let $\varepsilon_{i}: T \rightarrow \mathbb{C}^{*}$ be the character that maps a diagonal matrix to its $i$ th entry. We then have $\varepsilon_{n+1}=0$ and $\varepsilon_{i}+\varepsilon_{2 n+2-i}=0$ for $1 \leqslant i \leqslant 2 n+1$. The set of simple roots is $\Delta=\left\{\varepsilon_{1}-\varepsilon_{2}, \varepsilon_{2}-\varepsilon_{3}, \ldots, \varepsilon_{n-1}-\varepsilon_{n}, \varepsilon_{n}\right\}$, the unique cominuscule simple root is $\gamma=\varepsilon_{1}-\varepsilon_{2}$, and $\omega_{\gamma}=\varepsilon_{1}$. Let $\mathbb{C}\left[x_{1}, \ldots, x_{2 n+1}\right]$ be the coordinate ring of $E$, and set $q=x_{1} x_{2 n+1}+x_{2} x_{2 n}+\cdots+x_{n} x_{n+2}+\frac{1}{2} x_{n+1}^{2}$. The cominuscule variety $G / P$ corresponding to $\gamma$ can be identified with the quadric hypersurface $X=Z(q) \subset \mathbb{P} E$ of dimension 


\section{A Chevalley formula for equivariant quantum $K$-Theory}

$2 n-1$. The set $\mathcal{P}_{X}=\left\{\varepsilon_{1}-\varepsilon_{i} \mid 2 \leqslant i \leqslant 2 n\right\}$ is represented by the following diagram, where the boxes increase from left to right:

\begin{tabular}{|c|c|c|c|c|c|c|c|c|}
\hline$\gamma$ & $\varepsilon_{1}-\varepsilon_{3}$ & $\cdots$ & $\varepsilon_{1}-\varepsilon_{n}$ & $\varepsilon_{1}$ & $\varepsilon_{1}+\varepsilon_{n}$ & $\cdots$ & $\varepsilon_{1}+\varepsilon_{3}$ & $\varepsilon_{1}+\varepsilon_{2}$ \\
\hline
\end{tabular}

The $T$-fixed points of $X$ are the points $\left\langle e_{k}\right\rangle=\mathbb{C} e_{k}$ for $1 \leqslant k \leqslant 2 n+1$ and $k \neq n+1$. For $k \in[0,2 n-1]$, we let $X^{k}$ denote the $B^{-}$-stable Schubert variety of codimension $k$ in $X$. More precisely, we have $X^{k}=\overline{B^{-} \cdot\left\langle e_{k+1}\right\rangle}=Z\left(x_{1}, \ldots, x_{k}, q\right)$ for $0 \leqslant k \leqslant n-1$ and $X^{k}=\overline{B^{-} \cdot\left\langle e_{k+2}\right\rangle}=$ $Z\left(x_{1}, \ldots, x_{k+1}\right)$ for $n \leqslant k \leqslant 2 n-1$. Set $\mathcal{O}^{k}=\left[\mathcal{O}_{X^{k}}\right]$, and let $J_{k} \in K^{T}$ (point) be the restriction of $J$ to the $T$-fixed point defining $X^{k}$. Since $\varepsilon_{1}$ is the only short box in $\mathcal{P}_{X}$, it follows from Proposition 4.2 that the Chevalley formula $J \cdot \mathcal{O}^{k}=J_{k} \theta_{0}\left(\phi\left(\mathcal{O}^{k}\right)\right)$ holds whenever $k \neq n-1$.

Let $\iota: X \rightarrow \mathbb{P} E$ be the inclusion, and set $\widehat{J}=\left[\mathbb{C}_{-\varepsilon_{1}} \otimes \mathcal{O}_{\mathbb{P} E}(-1)\right] \in K^{T}(\mathbb{P} E)$. Then $\iota^{*}(\widehat{J})=J$ in $K^{T}(X)$. Since the pushforward map $\iota_{*}: K^{T}(X) \rightarrow K^{T}(\mathbb{P} E)$ is injective, it is enough to show that the identity $\widehat{J} \cdot \mathcal{O}^{n-1}=J_{n-1} \theta_{0}\left(\phi\left(\mathcal{O}^{n-1}\right)\right)$ holds in $K^{T}(\mathbb{P} E)$. Since $\varepsilon_{n}$ is the only short simple root, we deduce that $\delta\left(\varepsilon_{1}\right)=\varepsilon_{n}$ and $\phi\left(\mathcal{O}^{n-1}\right)=\mathcal{O}^{n-1}-\left[\mathbb{C}_{-\varepsilon_{n}}\right] \mathcal{O}^{n}$. Using that $\left[\mathcal{O}_{Z\left(x_{i}\right)}\right]=1-\left[\mathbb{C}_{-\varepsilon_{i}}\right]\left[\mathcal{O}_{\mathbb{P} E}(-1)\right]$ in $K^{T}(\mathbb{P} E)$ for each $i$, we also have

$$
\begin{aligned}
{\left[\mathcal{O}_{Z(q)}\right] } & =1-\left[\mathcal{O}_{\mathbb{P} E}(-2)\right]=1-\left[\mathcal{O}_{\mathbb{P} E}(-1)\right]+\left[\mathcal{O}_{\mathbb{P} E}(-1)\right] \cdot\left[\mathcal{O}_{Z\left(x_{n+1}\right)}\right] \\
& =\left[\mathcal{O}_{Z\left(x_{n+1}\right)}\right]+\left[\mathbb{C}_{-\varepsilon_{n}}\right]\left(1-\left[\mathcal{O}_{Z\left(x_{n+2}\right)}\right]\right) \cdot\left[\mathcal{O}_{Z\left(x_{n+1}\right)}\right] \\
& =\left[\mathcal{O}_{Z\left(x_{n+1}\right)}\right]+\left[\mathbb{C}_{-\varepsilon_{n}}\right]\left[\mathcal{O}_{Z\left(x_{n+1}\right)}\right]-\left[\mathbb{C}_{-\varepsilon_{n}}\right]\left[\mathcal{O}_{Z\left(x_{n+1}, x_{n+2}\right)}\right] .
\end{aligned}
$$

Since $X^{n-1}=Z\left(x_{1}, \ldots, x_{n-1}\right) \cap Z(q)$ and $J_{n-1}=\left.\widehat{J}\right|_{\left\langle e_{n}\right\rangle}=\left[\mathbb{C}_{\varepsilon_{n}-\varepsilon_{1}}\right]$, we obtain

$$
\begin{aligned}
\widehat{J} \cdot \mathcal{O}^{n-1} & =J_{n-1}\left(1-\left[\mathcal{O}_{Z\left(x_{n}\right)}\right]\right) \cdot \mathcal{O}^{n-1}=J_{n-1} \mathcal{O}^{n-1}-J_{n-1}\left[\mathcal{O}_{Z\left(x_{1}, \ldots, x_{n}\right)}\right] \cdot\left[\mathcal{O}_{Z(q)}\right] \\
& =J_{n-1} \mathcal{O}^{n-1}-J_{n-1}\left(\mathcal{O}^{n}+\left[\mathbb{C}_{-\varepsilon_{n}}\right] \mathcal{O}^{n}-\left[\mathbb{C}_{-\varepsilon_{n}}\right] \mathcal{O}^{n+1}\right)=J_{n-1} \theta_{0}\left(\phi\left(\mathcal{O}^{n-1}\right)\right) .
\end{aligned}
$$

This completes the proof of Theorem 3.6.

\section{Solutions to the Molev-Sagan equations}

\subsection{Recursive identities}

In this section, we let $X=G / P$ be an arbitrary flag manifold and show that the $T$-equivariant quantum $K$-theory ring $\mathrm{QK}_{T}(X)$, together with its Schubert structure constants and the underlying (three-point, genus zero) Gromov-Witten invariants of $X$, is uniquely determined by the products that involve divisor classes. Here $T$ denotes a maximal torus in $G$.

It was observed by Molev and Sagan [MS99] that the multiplicative structure constants of factorial Schur polynomials satisfy recursive identities that we will call Molev-Sagan equations. Related properties of factorial Schur polynomials had previously been studied by Okounkov and Olshanski [Oko96, O098]. Knutson and Tao used the Molev-Sagan equations to study the equivariant Schubert structure constants of Grassmannians [KT03]. In this context, the identities follow from the Chevalley formula together with the fact that the equivariant cohomology ring is associative. It was shown in [Mih07] that the same method can be applied to arbitrary flag varieties. Molev-Sagan-type equations have by now been used to prove Littlewood-Richardson rules in several papers [MS99, KT03, Buc15, PY17].

The Molev-Sagan equations provide a triangular system of identities that reduce arbitrary equivariant Schubert structure constants $C_{u, v}^{w}$ to the special constants of the form $C_{w, w}^{w}$, which are given by a simple formula of Kostant and Kumar [KK86]. Mihalcea's Chevalley formula 


\section{A.S. Buch, P.-E. Chaput, L.C. Mihalcea and N. Perrin}

[Mih07] for the equivariant quantum cohomology ring $\mathrm{QH}_{T}(X)$ can be used in a similar way to produce equations satisfied by the equivariant Gromov-Witten invariants of $X$, but in this case the equations are no longer triangular, and no analogue of the formula for $C_{w, w}^{w}$ is known. This added complexity was handled in [Mih06a, Mih07] with more refined recursive identities that reduce all (three-point, genus zero) equivariant Gromov-Witten invariants to those associated with multiplication with the identity class. This leads to the surprising realization that, even though the ring $\mathrm{QH}_{T}(X)$ may not be generated by divisor classes, the structure of this ring is completely determined by its Chevalley formula, and the same conclusion holds for the equivariant cohomology ring $H_{T}^{*}(X ; \mathbb{Z}){ }^{1}$

In this section, we show that the equivariant quantum $K$-theory ring $\mathrm{QK}_{T}(X)$ is similarly determined by multiplication with divisor classes, despite the fact that a formula for multiplication with divisors is known only when $X$ is cominuscule. We will derive this result as a formal consequence of the same property of the equivariant cohomology ring $H_{T}^{*}(X ; \mathbb{Z})$. For this reason, we start by considering this case, where we reprove and slightly extend some results from [Mih07] in a form that will be useful later. This part of our analysis is also valid for the equivariant cohomology of any Kac-Moody flag variety; see Remark 5.9.

\subsection{Equivariant cohomology}

Let $\Lambda=H_{T}^{*}$ (point; $\left.\mathbb{Z}\right)$ denote the equivariant cohomology of a point, and recall the notation from Section 2. The ring $H_{T}^{*}(X ; \mathbb{Z})$ is a free $\Lambda$-module with a basis of the Schubert classes $\left[X^{u}\right]$ for $u \in W^{P}$. The equivariant Schubert structure constants of $X$ are the classes $C_{u, v}^{w} \in \Lambda$, defined for $u, v, w \in W^{P}$ by the identity

$$
\left[X^{u}\right] \cdot\left[X^{v}\right]=\sum_{w} C_{u, v}^{w}\left[X^{w}\right]
$$

in $H_{T}^{*}(X ; \mathbb{Z})$. The constant $C_{u, v}^{w}$ is non-zero only if $u \leqslant w$ and $v \leqslant w$ in the Bruhat order of $W^{P}$.

For any character $\lambda: T \rightarrow \mathbb{C}^{*}$, we set $c_{T}(\lambda)=c_{1}\left(\mathbb{C}_{\lambda}\right) \in \Lambda$. The ring $\Lambda$ is generated by these classes, and the classes $c_{T}(\beta)$ for $\beta \in \Delta$ are algebraically independent. A class in $\Lambda$ is non-negative if it can be written as a polynomial with non-negative integer coefficients in the classes $c_{T}(\beta)$ for $\beta \in \Delta$. Graham has proved that all equivariant structure constants $C_{u, v}^{w}$ are non-negative classes [Gra01]. For $\beta \in \Delta$, we let $\omega_{\beta}$ denote the corresponding fundamental weight. Then the Chevalley formula for $H_{T}^{*}(X ; \mathbb{Z})\left[\right.$ Che94, KK86] states that for $\beta \in \Delta \backslash \Delta_{P}$ and $u, w \in W^{P}$, we have

$$
C_{s_{\beta}, u}^{w}= \begin{cases}c_{T}\left(\omega_{\beta}-u \cdot \omega_{\beta}\right) & \text { if } w=u, \\ \left(\omega_{\beta}, \alpha^{\vee}\right) & \text { if } \ell(w)=\ell(u)+1 \text { and } \exists \text { a } \alpha \in \Phi^{+} \text {with } w=u s_{\alpha}, \\ 0 & \text { otherwise }\end{cases}
$$

The following was proved in [Mih07]. We summarize the proof for completeness.

Lemma 5.1. Let $u, w \in W^{P}$ be minimal representatives.

(a) If $u \leqslant w$, then $C_{s_{\beta}, w}^{w}-C_{s_{\beta}, u}^{u}$ is a non-negative class in $\Lambda$ for every $\beta \in \Delta \backslash \Delta_{P}$.

(b) If $u \neq w$, then there exists a $\beta \in \Delta \backslash \Delta_{P}$ such that $C_{s_{\beta}, w}^{w} \neq C_{s_{\beta}, u}^{u}$.

(c) If $u<w$ and $C_{s_{\beta}, w}^{w} \neq C_{s_{\beta}, u}^{u}$, then there exists a $u^{\prime} \in W^{P}$ such that $u<u^{\prime} \leqslant w, \ell\left(u^{\prime}\right)=$ $\ell(u)+1$, and $C_{s_{\beta}, u}^{u^{\prime}} \neq 0$.

\footnotetext{
${ }^{1}$ After this paper was finished, we learned from Ciocan-Fontanine that this can be explained using a localization argument. See Remark 5.10 for details.
} 


\section{A Chevalley formula for equivariant quantum $K$-Theory}

Proof. If $u \neq w$, then choose $\beta \in \Delta \backslash \Delta_{P}$ such that $s_{\beta}$ occurs in a reduced expression for $u^{-1} w$. Then it follows from [Hum90, Theorem 1.12] that $u \cdot \omega_{\beta} \neq w \cdot \omega_{\beta}$. This proves part (b). Assume now that $u<w$. Choose $v \in W^{P}$ such that $u<v \leqslant w$ and $\ell(v)=\ell(u)+1$, and choose $\alpha \in \Phi^{+}$ such that $v=u s_{\alpha}$. Then $u . \alpha \in \Phi^{+}$. For any $\beta \in \Delta \backslash \Delta_{P}$, we have $u s_{\alpha} \cdot \omega_{\beta}=u$. $\left(\omega_{\beta}-\left(\omega_{\beta}, \alpha^{\vee}\right) \alpha\right)=$ $u . \omega_{\beta}-\left(\omega_{\beta}, \alpha^{\vee}\right) u . \alpha$, so $C_{s_{\beta}, v}^{v}-C_{s_{\beta}, u}^{u}=c_{T}\left(u \cdot \omega_{\beta}-v \cdot \omega_{\beta}\right)=\left(\omega_{\beta}, \alpha^{\vee}\right) c_{T}(u . \alpha)$ is a non-negative class in $\Lambda$. Part (a) follows from this by induction on $\ell(w)-\ell(u)$. Notice also that, when $u<v$ and $\ell(v)=\ell(u)+1$, we have $C_{s_{\beta}, u}^{v} \neq 0$ if and only if $u \cdot \omega_{\beta} \neq v \cdot \omega_{\beta}$. To prove part (c), assume that $u \cdot \omega_{\beta}=v \cdot \omega_{\beta} \neq w \cdot \omega_{\beta}$. By induction on $\ell(w)-\ell(u)$, we can find a $v^{\prime} \in W^{P}$ such that $v<v^{\prime} \leqslant w$ and $C_{s_{\beta}, v}^{v^{\prime}} \neq 0$. By part (b), we may choose $\gamma \in \Delta \backslash \Delta_{P}$ with $C_{s_{\gamma}, u}^{v} \neq 0$. This implies that $\left[X^{v^{\prime}}\right]$ occurs with non-zero coefficient in the product $\left[X^{s_{\beta}}\right] \cdot\left[X^{s_{\gamma}}\right] \cdot\left[X^{u}\right]$ in the ordinary cohomology ring $H^{*}(X ; \mathbb{Z})$. Since this ring is associative, we deduce that $C_{s_{\beta}, u}^{u^{\prime}} \neq 0$ for some $u^{\prime} \in W^{P}$ with $u<u^{\prime}<v^{\prime}$.

Let $\Lambda_{0}$ denote the field of fractions of $\Lambda=H_{T}^{*}$ (point; $\mathbb{Z}$ ). We will say that a class in $\Lambda_{0}$ is rationally positive if it can be written as a quotient of non-zero non-negative classes in $\Lambda$. All rationally positive classes are non-zero, and all sums, products, and quotients of rationally positive classes are again rationally positive. However, notice that some rationally positive classes in $\Lambda$ are not non-negative; for example, we have $x^{2}-x+1=\left(x^{3}+1\right) /(x+1)$.

Definition 5.2. Let $\left\{D_{u, v}^{w}\right\}$ be any vector of classes $D_{u, v}^{w} \in \Lambda_{0}$ indexed by triples $(u, v, w) \in$ $\left(W^{P}\right)^{3}$. We will say that this vector satisfies the (generalized) Molev-Sagan equations for $H_{T}^{*}(X ; \mathbb{Z})$ if for all $u, v, w \in W^{P}$ and $\beta \in \Delta \backslash \Delta_{P}$, we have

$$
\left(C_{s_{\beta}, w}^{w}-C_{s_{\beta}, u}^{u}\right) D_{u, v}^{w}=\sum_{u^{\prime}>u} C_{s_{\beta}, u}^{u^{\prime}} D_{u^{\prime}, v}^{w}-\sum_{w^{\prime}<w} C_{s_{\beta}, w^{\prime}}^{w} D_{u, v}^{w^{\prime}}
$$

and

$$
\left(C_{s_{\beta}, w}^{w}-C_{s_{\beta}, v}^{v}\right) D_{u, v}^{w}=\sum_{v^{\prime}>v} C_{s_{\beta}, v}^{v^{\prime}} D_{u, v^{\prime}}^{w}-\sum_{w^{\prime}<w} C_{s_{\beta}, w^{\prime}}^{w} D_{u, v}^{w^{\prime}}
$$

where the sums are over all elements $u^{\prime}, v^{\prime}$, or $w^{\prime}$ in $W^{P}$ with the indicated bounds.

The associativity relations $\left(\left[X^{s_{\beta}}\right] \cdot\left[X^{u}\right]\right) \cdot\left[X^{v}\right]=\left[X^{s_{\beta}}\right] \cdot\left(\left[X^{u}\right] \cdot\left[X^{v}\right]\right)$ and $\left[X^{u}\right] \cdot\left(\left[X^{v}\right] \cdot\right.$ $\left.\left[X^{s_{\beta}}\right]\right)=\left(\left[X^{u}\right] \cdot\left[X^{v}\right]\right) \cdot\left[X^{s_{\beta}}\right]$ of the ring $H_{T}^{*}(X ; \mathbb{Z})$ imply that the equivariant Schubert structure constants $\left\{C_{u, v}^{w}\right\}$ of $X$ form a solution to the Molev-Sagan equations. It is known that the Molev-Sagan equations together with known expressions [KK86] for the special coefficients $C_{w, w}^{w}$ uniquely determine these structure constants [MS99, KT03, Mih07]. It is interesting to note that every solution $\left\{D_{u, v}^{w}\right\}$ to the Molev-Sagan equations must satisfy the commutativity relation $D_{u, v}^{w}=D_{v, u}^{w}$, but we will not use this fact.

ExAmPle 5.3. Let $X=\mathbb{P}^{1}$ and $W^{P}=\{1, s\}$, and set $a=C_{s, s}^{s} \in \Lambda$. Then all solutions $\left\{D_{u, v}^{w}\right\}$ to the Molev-Sagan equations are given by

$$
D_{1, s}^{s}=D_{s, 1}^{s}=\frac{1}{a} D_{s, s}^{s}, \quad D_{1,1}^{s}=\frac{1}{a^{2}} D_{s, s}^{s}-\frac{1}{a} D_{1,1}^{1}, \quad \text { and } \quad D_{1, s}^{1}=D_{s, 1}^{1}=D_{s, s}^{1}=0,
$$

where $D_{1,1}^{1}$ and $D_{s, s}^{s}$ can be chosen freely in $\Lambda_{0}$. Every solution to the Molev-Sagan equations defines an associative algebra $H=\operatorname{Span}_{\Lambda_{0}}\left\{\sigma_{1}, \sigma_{s}\right\}$ with multiplication $\sigma_{u} \cdot \sigma_{v}=\sum_{w} D_{u, v}^{w} \sigma_{w}$. The Chevalley formula holds in $H$ if and only if $D_{s, s}^{s}=a$.

Proposition 5.4. There exist classes $A_{u, v}^{w}(\tau) \in \Lambda_{0}$, indexed by all quadruples $(u, v, w, \tau)$ in $\left(W^{P}\right)^{4}$, with the following properties: 


\section{A.S. Buch, P.-E. Chaput, L.C. Mihalcea and N. Perrin}

(a) Every solution $\left\{D_{u, v}^{w}\right\}$ to the Molev-Sagan equations for $H_{T}^{*}(X ; \mathbb{Z})$ satisfies

$$
D_{u, v}^{w}=\sum_{\tau \in W^{P}} A_{u, v}^{w}(\tau) D_{\tau, \tau}^{\tau} .
$$

(b) The class $A_{u, v}^{w}(\tau)$ is non-zero only if $u \leqslant w, v \leqslant w$, and $\tau \leqslant w$.

(c) If $u \leqslant w$ and $v \leqslant w$, then $A_{u, v}^{w}(w)$ is rationally positive.

Proof. Let $(u, v, w) \in\left(W^{P}\right)^{3}$ be any triple. Assume by induction that $A_{u^{\prime}, v^{\prime}}^{w^{\prime}}(\tau)$ has been defined for all triples $\left(u^{\prime}, v^{\prime}, w^{\prime}\right)$ for which $w^{\prime}<w$, or $w^{\prime}=w$ and $v^{\prime}>v$, or $w^{\prime}=w$ and $v^{\prime}=v$ and $u^{\prime}>u$, and for all $\tau \in W^{P}$. Assume also that these classes satisfy properties (a), (b), and (c). We define the classes $A_{u, v}^{w}(\tau)$ for $\tau \in W^{P}$ as follows.

Assume first that $v \neq w$. According to Lemma 5.1(b), we may choose $\beta \in \Delta \backslash \Delta_{P}$ such that $C_{s_{\beta}, w}^{w} \neq C_{s_{\beta}, v}^{v}$. Using this choice, we define

$$
A_{u, v}^{w}(\tau)=\frac{1}{C_{s_{\beta}, w}^{w}-C_{s_{\beta}, v}^{v}}\left(\sum_{v^{\prime}>v} C_{s_{\beta}, v}^{v^{\prime}} A_{u, v^{\prime}}^{w}(\tau)-\sum_{w^{\prime}<w} C_{s_{\beta}, w^{\prime}}^{w} A_{u, v}^{w^{\prime}}(\tau)\right) .
$$

If $u \neq v=w$, then choose $\beta \in \Delta \backslash \Delta_{P}$ such that $C_{s_{\beta}, w}^{w} \neq C_{s_{\beta}, u}^{u}$ and define

$$
A_{u, w}^{w}(\tau)=\frac{1}{C_{s_{\beta}, w}^{w}-C_{s_{\beta}, u}^{u}} \sum_{u^{\prime}>u} C_{s_{\beta}, u}^{u^{\prime}} A_{u^{\prime}, w}^{w}(\tau) .
$$

Finally, if $u=v=w$, then define $A_{w, w}^{w}(\tau)=\delta_{w, \tau}$.

We must check that the classes $A_{u, v}^{w}(\tau)$ satisfy properties (a), (b), and (c). These properties are clear if $u=v=w$. In addition, property (b) follows immediately from the definition of $A_{u, v}^{w}(\tau)$ together with the induction hypothesis. Assume that $v \neq w$. Then property (a) holds because

$$
\begin{aligned}
\left(C_{s_{\beta}, w}^{w}-C_{s_{\beta}, v}^{v}\right) D_{u, v}^{w} & =\sum_{v^{\prime}>v} C_{s_{\beta}, v}^{v^{\prime}} D_{u, v^{\prime}}^{w}-\sum_{w^{\prime}<w} C_{s_{\beta}, w^{\prime}}^{w} D_{u, v}^{w^{\prime}} \\
& =\sum_{v^{\prime}>v} C_{s_{\beta}, v}^{v^{\prime}} \sum_{\tau} A_{u, v^{\prime}}^{w}(\tau) D_{\tau, \tau}^{\tau}-\sum_{w^{\prime}<w} C_{s_{\beta}, w^{\prime}}^{w} \sum_{\tau} A_{u, v}^{w^{\prime}}(\tau) D_{\tau, \tau}^{\tau} \\
& =\sum_{\tau}\left(\sum_{v^{\prime}>v} C_{s_{\beta}, v}^{v^{\prime}} A_{u, v^{\prime}}^{w}(\tau)-\sum_{w^{\prime}<w} C_{s_{\beta}, w^{\prime}}^{w} A_{u, v}^{w^{\prime}}(\tau)\right) D_{\tau, \tau}^{\tau} \\
& =\left(C_{s_{\beta}, w}^{w}-C_{s_{\beta}, v}^{v}\right) \sum_{\tau} A_{u, v}^{w}(\tau) D_{\tau, \tau}^{\tau} .
\end{aligned}
$$

In addition, if $u \leqslant w$ and $v<w$, then it follows from property (b) that

$$
A_{u, v}^{w}(w)=\frac{1}{C_{s_{\beta}, w}^{w}-C_{s_{\beta}, v}^{v}} \sum_{v^{\prime}>v} C_{s_{\beta}, v}^{v^{\prime}} A_{u, v^{\prime}}^{w}(w),
$$

and parts (a) and (c) of Lemma 5.1 together with property (c) of the induction hypothesis imply that this sum is rationally positive. A similar argument shows that properties (a) and (c) hold when $u \neq v=w$.

Corollary 5.5. Let $\left\{D_{u, v}^{w}\right\}$ be any solution to the Molev-Sagan equations for the ring $H_{T}^{*}(X ; \mathbb{Z})$ that also satisfies the additional condition

$$
\forall w \in W^{P} \exists u, v \in W^{P} \text { such that } u \leqslant w \text { and } v \leqslant w \text { and } D_{u, v}^{w}=C_{u, v}^{w} .
$$

Then we have $D_{u, v}^{w}=C_{u, v}^{w}$ for all $u, v, w \in W^{P}$. 


\section{A Chevalley formula for equivariant quantum $K$-Theory}

Proof. In view of Proposition 5.4, it is enough to show that $D_{\tau, \tau}^{\tau}=C_{\tau, \tau}^{\tau}$ for all $\tau \in W^{P}$. Let $w \in W^{P}$, and assume by induction that $D_{\tau, \tau}^{\tau}=C_{\tau, \tau}^{\tau}$ for all $\tau<w$. Choose $u, v \in W^{P}$ such that $u \leqslant w, v \leqslant w$, and $D_{u, v}^{w}=C_{u, v}^{w}$. Then we have

$$
A_{u, v}^{w}(w) D_{w, w}^{w}=D_{u, v}^{w}-\sum_{\tau<w} A_{u, v}^{w}(\tau) D_{\tau, \tau}^{\tau}=C_{u, v}^{w}-\sum_{\tau<w} A_{u, v}^{w}(\tau) C_{\tau, \tau}^{\tau}=A_{u, v}^{w}(w) C_{w, w}^{w} .
$$

Since $A_{u, v}^{w}(w) \neq 0$ by Proposition 5.4(c), this implies that $D_{w, w}^{w}=C_{w, w}^{w}$.

Remark 5.6. The following instances of the additional condition of Corollary 5.5 have appeared: $D_{w, w}^{w}=C_{w, w}^{w}$ in [MS99, KT03], $D_{w, 1}^{w}=1$ in [Mih07], and $D_{1,1}^{w}=\delta_{1, w}$ in [Mih07].

Corollary 5.7. Let $H$ be any associative $\Lambda$-algebra that is also a free $\Lambda$-module with a basis $\left\{\sigma_{u} \mid u \in W^{P}\right\}$ indexed by $W^{P}$. Assume that $H$ satisfies the (two-sided) Chevalley formula

$$
\sigma_{s_{\beta}} \cdot \sigma_{u}=\sigma_{u} \cdot \sigma_{s_{\beta}}=\sum_{w \in W^{P}} C_{s_{\beta}, u}^{w} \sigma_{w}
$$

for all $u \in W^{P}$ and $\beta \in \Delta \backslash \Delta_{P}$, as well as the identity $\sigma_{1} \cdot \sigma_{1}=\sigma_{1}$. Then the homomorphism of $\Lambda$-modules $H \rightarrow H_{T}^{*}(X ; \mathbb{Z})$ defined by $\sigma_{u} \mapsto\left[X^{u}\right]$ is an isomorphism of rings.

Proof. Let $\left\{D_{u, v}^{w}\right\}$ be the structure constants of $H$ with respect to the basis $\left\{\sigma_{u}\right\}$. Then the relations $\left(\sigma_{s_{\beta}} \cdot \sigma_{u}\right) \cdot \sigma_{v}=\sigma_{s_{\beta}} \cdot\left(\sigma_{u} \cdot \sigma_{v}\right)$ and $\sigma_{u} \cdot\left(\sigma_{v} \cdot \sigma_{s_{\beta}}\right)=\left(\sigma_{u} \cdot \sigma_{v}\right) \cdot \sigma_{s_{\beta}}$ imply that $\left\{D_{u, v}^{w}\right\}$ is a solution to the Molev-Sagan equations. The identity $\sigma_{1} \cdot \sigma_{1}=\sigma_{1}$ implies that $D_{1,1}^{w}=\delta_{w, 1}=C_{1,1}^{w}$ for all $w \in W^{P}$. It therefore follows from Corollary 5.5 that $D_{u, v}^{w}=C_{u, v}^{w}$ for all $u, v, w \in W^{P}$, as required.

The following conjecture implies that the vector space of all solutions to the Molev-Sagan equations has dimension equal to the cardinality of $W^{P}$.

Conjecture 5.8. The classes $A_{u, v}^{w}(\tau)$ of Proposition 5.4 are uniquely determined by property (a). Equivalently, for each fixed $\tau \in W^{P}$, the vector $\left\{A_{u, v}^{w}(\tau)\right\}$ is the unique solution to the Molev-Sagan equations that also satisfies $A_{w, w}^{w}(\tau)=\delta_{w, \tau}$ for all $w \in W^{P}$.

Remark 5.9. The results proved in Section 5.2 are true also when $X=G / P$ is a homogeneous space defined by a Kac-Moody group $G$ and a parabolic subgroup $P$, with the same proofs. The required Chevalley formula for the equivariant cohomology of such spaces is proved in Kumar's book [Kum02, Theorem 11.1.7].

Remark 5.10. Ciocan-Fontanine brought to our attention that, under certain hypotheses, [CKS08, Lemma 4.1.3] implies that the localized equivariant cohomology $\operatorname{ring} H_{T}^{*}(X ; \mathbb{Z}) \otimes_{\Lambda}$ $\Lambda_{0}$ is generated by divisors. We next give an alternative proof of this conclusion. Let $D=$ $\sum_{\beta \in \Delta \backslash \Delta_{P}}\left[X^{s_{\beta}}\right]$ be the sum of the Schubert divisors in $X$.

Lemma 5.11. The ring $H_{T}^{*}(X ; \mathbb{Z}) \otimes_{\Lambda} \Lambda_{0}$ is generated by $D$ as a $\Lambda_{0}$-algebra.

Proof. Consider the $\Lambda$-linear endomorphism $\mu_{D}: H_{T}^{*}(X ; \mathbb{Z}) \rightarrow H_{T}^{*}(X ; \mathbb{Z})$ defined as multiplication by $D$. Since multiplication by $D$ can be written in the form $D \cdot\left[X^{u}\right]=D_{u}\left[X^{u}\right]+$ $\sum_{w>u} c(u ; w)\left[X^{w}\right]$, where $c(u ; w) \in \Lambda$ and $D_{u}$ is the restriction of $D$ to the $T$-fixed point $u . P$, it follows that $\mu_{D}$ is represented by an upper-triangular matrix. The diagonal entries are given by $D_{u}=c_{T}(\omega-u . \omega)$, where $\omega=\sum_{\beta \in \Delta \backslash \Delta_{P}} \omega_{\beta}$ (see [KK90] or, for example, [BM15, Theorem 8.1]). Since we have $v \cdot \omega=\omega$ if and only if $v \in W_{P}$ [Bou68, V, Section 4.6], we deduce that $\mu_{D}$ has 


\section{A.S. Buch, P.-E. Chaput, L.C. Mihalcea And N. Perrin}

distinct diagonal entries. This implies that the minimal polynomial of $\mu_{D}$ is equal to the characteristic polynomial, so the elements $1, D, D^{2}, \ldots, D^{\# W_{P}-1}$ are linearly independent over $\Lambda_{0}$ and $\operatorname{span} H_{T}^{*}(X ; \mathbb{Z}) \otimes_{\Lambda} \Lambda_{0}$.

In relation to [CKS08], consider the $T$-equivariant embedding $X \subset \mathbb{P}(V)$, where $V=$ $H^{0}\left(X, \mathcal{O}_{X}(D)\right)$. In order to apply [CKS08, Lemma 4.1.3] to this situation, one must show that the restriction map $H^{*}\left(\mathbb{P}(V)^{T} ; \mathbb{C}\right) \rightarrow H^{*}\left(X^{T} ; \mathbb{C}\right)=\mathbb{C}^{\# W^{P}}$ is surjective. In fact, this follows from Lemma 5.11 together with the commutative diagram used in the proof of [CKS08, Lemma 4.1.3]. However, it is not in general true that $\mathbb{P}(V)$ has isolated $T$-fixed points. For example, this fails when $X$ is an adjoint flag variety in the sense that $V=\operatorname{Lie}(G)$ is the adjoint representation of $G$; see, for example, [CP11a].

\subsection{Equivariant quantum $K$-theory}

We finally show that the ring $\mathrm{QK}_{T}(X)$ is uniquely determined by multiplication with the divisor classes and the identity element. Our argument is based on linear algebra and could also be applied to the equivariant $K$-theory $\operatorname{ring} K^{T}(X)$ or the equivariant quantum cohomology ring $\mathrm{QH}_{T}(X)$. For $u, v, w \in W^{P}$, we define the power series

$$
N_{u, v}^{w}=\sum_{d \geqslant 0} N_{u, v}^{w, d} q^{d}
$$

in the ring $\Gamma \llbracket q \rrbracket=\Gamma \llbracket q_{\beta} \mid \beta \in \Delta \backslash \Delta_{P} \rrbracket$. The product in $\mathrm{QK}_{T}(X)$ is given by

$$
\mathcal{O}^{u} \star \mathcal{O}^{v}=\sum_{w \in W^{P}} N_{u, v}^{w} \mathcal{O}^{w}
$$

Let $\Gamma \llbracket q \rrbracket_{0}$ be the field of fractions of $\Gamma \llbracket q \rrbracket$.

DeFinition 5.12. Let $\left\{D_{u, v}^{w}\right\}$ be a vector of classes $D_{u, v}^{w} \in \Gamma \llbracket q \rrbracket_{0}$ indexed by triples $(u, v, w) \in$ $\left(W^{P}\right)^{3}$. We say that this vector satisfies the generalized Molev-Sagan equations for $\mathrm{QK}_{T}(X)$ if for all $u, v, w \in W^{P}$ and $\beta \in \Delta \backslash \Delta_{P}$, we have

$$
\sum_{u^{\prime} \in W^{P}} N_{s_{\beta}, u}^{u^{\prime}} D_{u^{\prime}, v}^{w}=\sum_{w^{\prime} \in W^{P}} N_{s_{\beta}, w^{\prime}}^{w} D_{u, v}^{w^{\prime}}=\sum_{v^{\prime} \in W^{P}} N_{s_{\beta}, v}^{v^{\prime}} D_{u, v^{\prime}}^{w}
$$

The equalities $\left(\mathcal{O}^{s_{\beta}} \star \mathcal{O}^{u}\right) \star \mathcal{O}^{v}=\mathcal{O}^{s_{\beta}} \star\left(\mathcal{O}^{u} \star \mathcal{O}^{v}\right)=\mathcal{O}^{u} \star\left(\mathcal{O}^{s_{\beta}} \star \mathcal{O}^{v}\right)$ imply that the structure constants $\left\{N_{u, v}^{w}\right\}$ form a solution to the Molev-Sagan equations for $\mathrm{QK}_{T}(X)$. Notice that if each structure constant $N_{s_{\beta}, u}^{w}$ in Definition 5.12 is replaced with $C_{s_{\beta}, u}^{w}$, then the Molev-Sagan equations for $\mathrm{QK}_{T}(X)$ turn into the Molev-Sagan equations for $H_{T}^{*}(X ; \mathbb{Z})$. One noteworthy difference between the two cases is that the structure constants $N_{u, v}^{w}$ of $\mathrm{QK}_{T}(X)$ may be nonzero when $u \nless w$ and $v \nless w$. For this reason, the Molev-Sagan equations for $\operatorname{QK}_{T}(X)$ are not triangular, which makes it time-consuming to solve them. Another obstruction to solving the equations is that the constants $N_{s_{\beta}, u}^{w}$ are known only when $X$ is a cominuscule variety. Here, we will only discuss how the ring $\mathrm{QK}_{T}(X)$ is determined by the Molev-Sagan equations. A systematic method for solving the Molev-Sagan equations for the equivariant quantum cohomology ring $\mathrm{QH}_{T}(X)$ can be found in [Mih07].

Proposition 5.13. Let $\left\{D_{u, v}^{w}\right\}$ be any solution to the Molev-Sagan equations for $\mathrm{QK}_{T}(X)$ that also satisfies the additional condition

$$
\forall w \in W^{P} \exists u, v \in W^{P} \text { such that } u \leqslant w \text { and } v \leqslant w \text { and } D_{u, v}^{w}=N_{u, v}^{w} .
$$

Then we have $D_{u, v}^{w}=N_{u, v}^{w}$ for all $u, v, w \in W^{P}$. 


\section{A Chevalley formula for equivariant quantum $K$-Theory}

Before we prove Proposition 5.13, we first recall some facts about the equivariant $K$-theory ring $K^{T}(X)$. Let $\operatorname{ch}: \Gamma \rightarrow \widehat{\Lambda}:=\prod_{k=0}^{\infty} H_{T}^{2 k}(X ; \mathbb{Q})$ denote the equivariant Chern character from $[\mathrm{EG} 00, \S 3.1]$. This injective ring homomorphism is defined by $\operatorname{ch}\left(\left[\mathbb{C}_{\lambda}\right]\right)=\exp \left(c_{T}(\lambda)\right)$. For $\sigma \in$ $K^{T}(X)$, we may write $\operatorname{ch}(\sigma)=\sum \operatorname{ch}_{m}(\sigma)$, where $\operatorname{ch}_{m}(\sigma) \in H_{T}^{2 m}(X ; \mathbb{Q})$ is the term of degree $m$. Set $\operatorname{ch}_{m}(\sigma)=0$ for $m<0$. We will say that $\sigma$ has degree at least $k$ if $\operatorname{ch}_{m}(\sigma)=0$ for all $m<k$. If $\sigma \neq 0$, then the leading term of $\sigma$ is the first non-zero term $\operatorname{ch}_{m}(\sigma)$ (with $m$ minimal). For $u, v, w \in$ $W^{P}$, we set $d(u, v, w)=\ell(u)+\ell(v)-\ell(w)$. The structure constant $N_{u, v}^{w, 0}$ of $K^{T}(X)$ has degree at least $d(u, v, w)$, and we have $\operatorname{ch}_{d(u, v, w)}\left(N_{u, v}^{w, 0}\right)=C_{u, v}^{w}$. This can be proved geometrically using the equivariant Riemann-Roch formula [EG00, Corollary 3.1] (see [BM11, § 4.1]) or combinatorially using the Chevalley formula for $K^{T}(G / B)$ [LP07].

Proof of Proposition 5.13. According to Corollary 5.5, the vector $\left\{C_{u, v}^{w}\right\}$ of structure constants of $H_{T}^{*}(X ; \mathbb{Z})$ is the unique solution to the equations of Definition 5.2 together with a choice of $k=\# W^{P}$ additional equations of the form $D_{u, v}^{w}=C_{u, v}^{w}$. Since this is a system of linear equations, we can choose a subset of $k^{3}$ of the equations that are sufficient to determine the vector $\left\{C_{u, v}^{w}\right\}$. Consider the corresponding set of equations for $\mathrm{QK}_{T}(X)$. More precisely, if the chosen equations for $\left\{C_{u, v}^{w}\right\}$ include the first (respectively, second) equation of Definition 5.2 for some $u, v, w \in W^{P}$ and $\beta \in \Delta \backslash \Delta_{P}$, then we use the first (respectively, second) equality of Definition 5.12 given by the same elements $u, v, w, \beta$. For each equation of the form $D_{u, v}^{w}=C_{u, v}^{w}$, we similarly use the corresponding equation $D_{u, v}^{w}=N_{u, v}^{w}$. We claim that the vector $\left\{N_{u, v}^{w}\right\}$ is the unique solution to these equations for $\mathrm{QK}_{T}(X)$. These equations can be stated in matrix form as $A D=b$, where $A$ is a $k^{3} \times k^{3}$ matrix and $b$ is a vector of length $k^{3}$, both with entries from $\Gamma \llbracket q \rrbracket$, and $D=\left\{D_{u, v}^{w}\right\}$ is the vector of indeterminates. Since $\left\{N_{u, v}^{w}\right\}$ satisfies the Molev-Sagan equations for $\mathrm{QK}_{T}(X)$, it suffices to show that the determinant of $A$ is not zero. For this, it is enough to show that $\operatorname{det}(\bar{A}) \neq 0$, where $\bar{A}$ is obtained from $A$ by substituting zero for all quantum parameters $q_{\beta}$. We will show that the leading term of $\operatorname{det}(\bar{A})$ is the determinant of the corresponding system of linear equations for $H_{T}(X ; \mathbb{Z})$.

Number the rows and columns of $A$ from 1 to $k^{3}$, and define integers $d_{i}$ and $d_{j}^{\prime}$ for $1 \leqslant i, j \leqslant k^{3}$ as follows. If the $i$ th row of $A$ represents an equation coming from Definition 5.12 for a triple $(u, v, w) \in\left(W^{P}\right)^{3}$, then set $d_{i}=d(u, v, w)+1$. If the $i$ th row of $A$ represents (the left-hand side of) an equation $D_{u, v}^{w}=N_{u, v}^{w}$, then set $d_{i}=d(u, v, w)$. Finally, if the $j$ th column of $A$ corresponds to the indeterminate $D_{u, v}^{w}$, then set $d_{j}^{\prime}=d(u, v, w)$. It follows by inspection of the Molev-Sagan equations that each entry $\bar{A}_{i j}$ of $\bar{A}$ has degree at least $d_{i}-d_{j}^{\prime}$. This implies that $\operatorname{det}(\bar{A})$ has degree at least $\sum_{i} d_{i}-\sum_{j} d_{j}^{\prime}$. Furthermore, if we replace each entry $\bar{A}_{i j}$ of $\bar{A}$ with $\mathrm{ch}_{d_{i}-d_{j}^{\prime}}\left(\bar{A}_{i j}\right)$, then the result is the matrix of coefficients of the original $k^{3}$ equations for the structure constants of $H_{T}^{*}(X ; \mathbb{Z})$. Since this system of linear equations is known to have a unique solution, we deduce that the term of degree $\sum d_{i}-\sum d_{j}^{\prime}$ in $\operatorname{ch}(\operatorname{det}(\bar{A}))$ is indeed non-zero. This completes the proof.

Corollary 5.14. Let $H$ be any associative algebra over $\Gamma \llbracket q \rrbracket$ that is also a free $\Gamma \llbracket q \rrbracket$-module with a basis $\left\{\sigma_{u} \mid u \in W^{P}\right\}$ indexed by $W^{P}$. Assume that $H$ satisfies

$$
\sigma_{s_{\beta}} \star \sigma_{u}=\sigma_{u} \star \sigma_{s_{\beta}}=\sum_{w \in W^{P}} N_{s_{\beta}, u}^{w} \sigma_{w}
$$

for all $u \in W^{P}$ and $\beta \in \Delta \backslash \Delta_{P}$, as well as the identity $\sigma_{1} \star \sigma_{1}=\sigma_{1}$. Then the homomorphism of $\Gamma \llbracket q \rrbracket$-modules $H \rightarrow \mathrm{QK}_{T}(X)$ defined by $\sigma_{u} \mapsto \mathcal{O}^{u}$ is an isomorphism of rings. 


\section{A.S. Buch, P.-E. Chaput, L.C. Mihalcea and N. Perrin}

Remark 5.15. Gorbounov and Korff have used ideas from integrable systems to construct an algebra called $q h^{*}(\operatorname{Gr}(m, n))$, together with a basis for this algebra that corresponds to the Schubert classes of the Grassmannian $\operatorname{Gr}(m, n)$ [GK17]. ${ }^{2}$ It follows from [GK17, Corollary 3.18] that this algebra satisfies a Chevalley formula that agrees with our Theorem 3.9. We therefore deduce from Corollary 5.14 that the algebra $q h^{*}(\operatorname{Gr}(m, n))$ has the same Schubert structure constants as the equivariant quantum $K$-theory ring $\mathrm{QK}_{T}(\operatorname{Gr}(m, n))$. This proves that the two algebras are isomorphic, which is Conjecture 1.2 in [GK17]. In particular, the presentation (Theorem 5.16) and Giambelli formula (Corollary 5.13) proved in [GK17] are valid for the equivariant quantum $K$-theory of Grassmannians. We note that the relation of $q h^{*}(\operatorname{Gr}(m, n))$ with the equivariant quantum cohomology ring $\mathrm{QH}_{T}(\mathrm{Gr}(m, n))$ and the (non-equivariant) quantum $K$-theory ring $\mathrm{QK}(\mathrm{Gr}(m, n))$ was established in [GK17] by using the structure theorems from [Mih07, BM11]. We were informed by E. Gonzalez and C. Woodward that in a paper in preparation, they have also obtained a geometric proof of the presentation of $\mathrm{QK}(\operatorname{Gr}(m, n))$, by writing the Grassmannian as a geometric invariant theory quotient of a space of matrices.

\section{ACKNOWLEDGEMENTS}

This project was started while the authors visited the University of Copenhagen during the Summer of 2014. We thank the Mathematics Department in Copenhagen for their hospitality and for providing a friendly and stimulating environment. We also thank Ionut Ciocan-Fontanine for bringing to our attention [CKS08, Lemma 4.1.3] and an anonymous referee for valuable suggestions.

\section{REFERENCES}

AC15 D. Anderson and L. Chen, Positivity of equivariant Gromov-Witten invariants, Math. Res. Lett. 22 (2015), no. 1, 1-9; https://doi.org/10.4310/MRL.2015.v22.n1.a1.

AGM11 D. Anderson, S. Griffeth, and E. Miller, Positivity and Kleiman transversality in equivariant $K$-theory of homogeneous spaces, J. Eur. Math. Soc. 13 (2011), no. 1, 57-84; https://doi. org/10.4171/JEMS/244.

AJS94 H.H. Andersen, J.C. Jantzen, and W. Soergel, Representations of quantum groups at a pth root of unity and of semisimple groups in characteristic p: independence of $p$, Astérisque 220 (1994), 321 pp.

BCMP13 A. S. Buch, P.-E. Chaput, L. C. Mihalcea, and N. Perrin, Finiteness of cominuscule quantum K-theory, Ann. Sci. Éc. Norm. Supér. (4) 46 (2013), no. 3, 477-494; https://doi.org/10. 24033/asens. 2194.

BCMP16 _ Rational connectedness implies finiteness of quantum K-theory, Asian J. Math. 20 (2016), no. 1, 117-122; https://doi.org/10.4310/AJM.2016.v20.n1.a5.

BCMP18_, Projected Gromov-Witten varieties in cominuscule spaces, Proc. Amer. Math. Soc., published online on 15 May 2018, https://doi.org/10.1090/proc/13839, to appear in print.

Bil97 S. C. Billey, Kostant polynomials and the cohomology ring for $G / B$, Proc. Nat. Acad. Sci. USA 94 (1997), no. 1, 29-32; https://doi.org/10.1073/pnas.94.1.29.

BKT03 A.S. Buch, A. Kresch, and H. Tamvakis, Gromov-Witten invariants on Grassmannians, J. Amer. Math. Soc. 16 (2003), no. 4, 901-915; https://doi.org/10.1090/ S0894-0347-03-00429-6.

\footnotetext{
${ }^{2}$ The parameter $\beta$ used in [GK17] must be replaced with -1 in order to match our notation.
} 


\section{A Chevalley formula for equivariant quantum $K$-Theory}

BM11 A.S. Buch and L.C. Mihalcea, Quantum K-theory of Grassmannians, Duke Math. J. 156 (2011), no. 3, 501-538; https://doi.org/10.1215/00127094-2010-218.

BM15 _ Curve neighborhoods of Schubert varieties, J. Differential Geom. 99 (2015), no. 2, 255-283; https://doi.org/10.4310/jdg/1421415563.

Bou68 N. Bourbaki, Éléments de mathématique. Fasc. XXXIV. Groupes et algèbres de Lie. Chapitre IV: Groupes de Coxeter et systèmes de Tits. Chapitre V: Groupes engendrés par des réflexions. Chapitre VI: Systèmes de racines, Actualités Sci. Indust., vol. 1337 (Hermann, Paris, 1968).

BR12 A.S. Buch and V. Ravikumar, Pieri rules for the K-theory of cominuscule Grassmannians, J. reine angew. Math. 668 (2012), 109-132; https://doi.org/10.1515/CRELLE.2011.135.

Bri02 M. Brion, Positivity in the Grothendieck group of complex flag varieties, J. Algebra 258 (2002), no. 1, 137-159; https://doi.org/10.1016/S0021-8693(02)00505-7.

BS16 A.S. Buch and M.J. Samuel, K-theory of minuscule varieties, J. reine angew. Math. 719 (2016), 133-171; https://doi.org/10.1515/crelle-2014-0051.

Buc03 A.S. Buch, Quantum cohomology of Grassmannians, Compos. Math. 137 (2003), no. 2, 227235; https://doi.org/10.1023/A:1023908007545.

Buc15 Mutations of puzzles and equivariant cohomology of two-step flag varieties, Ann. of Math. 182 (2015), no. 1, 173-220; https://doi.org/10.4007/annals.2015.182.1.4.

CG97 N. Chriss and V. Ginzburg, Representation theory and complex geometry (Birkhäuser Boston, Inc., Boston, MA, 1997); https://doi.org/10.1007/978-0-8176-4938-8.

Che94 C. Chevalley, Sur les décompositions cellulaires des espaces $G / B$, Algebraic Groups and their Generalizations: Classical Methods (University Park, PA, 1991), Proc. Sympos. Pure Math., vol. 56 (Amer. Math. Soc., Providence, RI, 1994), 1-23.

CKS08 I. Ciocan-Fontanine, B. Kim, and C. Sabbah, The abelian/nonabelian correspondence and Frobenius manifolds, Invent. Math. 171 (2008), no. 2, 301-343; https://doi.org/10.1007/ s00222-007-0082-x.

CMP08 P.-E. Chaput, L. Manivel, and N. Perrin, Quantum cohomology of minuscule homogeneous spaces, Transform. Groups 13 (2008), no. 1, 47-89; https://doi.org/10.1007/ s00031-008-9001-5.

CP11a P.-E. Chaput and N. Perrin, On the quantum cohomology of adjoint varieties, Proc. Lond. Math. Soc. 103 (2011), no. 2, 294-330; https://doi.org/10.1112/plms/pdq052.

CP11b L Rationality of some Gromov-Witten varieties and application to quantum $K$ theory, Commun. Contemp. Math. 13 (2011), no. 1, 67-90; https://doi.org/10.1142/ S0219199711004166.

EG00 D. Edidin and W. Graham, Riemann-Roch for equivariant Chow groups, Duke Math. J. 102 (2000), no. 3, 567-594; https://doi.org/10.1215/S0012-7094-00-10239-6.

FP97 W. Fulton and R. Pandharipande, Notes on stable maps and quantum cohomology (Algebraic Geometry - Santa Cruz 1995), Proc. Sympos. Pure Math., vol. 62 (Amer. Math. Soc., Providence, RI, 1997), 45-96; https://doi.org/10.1090/pspum/062.2/1492534.

Ful98 W. Fulton, Intersection theory, second ed., Ergeb. Math. Grenzgeb. (3), vol. 2 (Springer-Verlag, Berlin, 1998); https://doi.org/10.1007/978-1-4612-1700-8.

Giv00 A. Givental, On the WDVV equation in quantum K-theory, Michigan Math. J. 48 (2000), 295-304; https://doi.org/10.1307/mmj/1030132720.

GK17 V. Gorbounov and C. Korff, Quantum integrability and generalised quantum Schubert calculus, Adv. Math. 313 (2017), 282-356; https://doi.org/10.1016/j.aim.2017.03.030.

GK15 W. Graham and V. Kreiman, Excited Young diagrams, equivariant K-theory, and Schubert varieties, Trans. Amer. Math. Soc. 367 (2015), no. 9, 6597-6645; https://doi.org/10.1090/ S0002-9947-2015-06288-6. 


\section{A.S. Buch, P.-E. Chaput, L.C. Mihalcea and N. Perrin}

GKM98 M. Goresky, R. Kottwitz, and R. MacPherson, Equivariant cohomology, Koszul duality, and the localization theorem, Invent. Math. 131 (1998), no. 1, 25-83; https://doi.org/10.1007/ s002220050197.

GR04 S. Griffeth and A. Ram, Affine Hecke algebras and the Schubert calculus, European J. Combin. 25 (2004), no. 8, 1263-1283; https://doi.org/10.1016/j.ejc.2003.10.012.

Gra01 W. Graham, Positivity in equivariant Schubert calculus, Duke Math. J. 109 (2001), no. 3, 599-614; https://doi.org/10.1215/S0012-7094-01-10935-6.

Har77 R. Hartshorne, Algebraic geometry, Grad. Texts Math., vol. 52 (Springer-Verlag, New York Heidelberg, 1977); https://doi.org/10.1007/978-1-4757-3849-0.

Hum75 J.E. Humphreys, Linear algebraic groups, Grad. Texts Math., vol. 21 (Springer-Verlag, New York - Heidelberg, 1975); https://doi.org/10.1007/978-1-4684-9443-3.

Hum90 _ Reflection groups and Coxeter groups, Cambridge Stud. Adv. Math., vol. 29 (Cambridge Univ. Press, Cambridge, 1990); https://doi.org/10.1017/CB09780511623646.

IMT15 H. Iritani, T. Milanov, and V. Tonita, Reconstruction and convergence in quantum K-theory via difference equations, Int. Math. Res. Not. 2015 (2015), no. 11, 2887-2937; https://doi. org/10.1093/imrn/rnu026.

KK86 B. Kostant and S. Kumar, The nil Hecke ring and cohomology of G/P for a Kac-Moody group G, Adv. Math. 62 (1986), no. 3, 187-237; https://doi.org/10.1016/0001-8708(86) 90101-5.

KK90_ T-equivariant K-theory of generalized flag varieties, J. Differential Geom. 32 (1990), no. 2, 549-603; https://doi.org/10.4310/jdg/1214445320.

KLS13 A. Knutson, T. Lam, and D.E. Speyer, Positroid varieties: juggling and geometry, Compos. Math. 149 (2013), no. 10, 1710-1752; https://doi.org/10.1112/S0010437X13007240.

KLS14 _ Projections of Richardson varieties, J. reine angew. Math. 687 (2014), 133-157; https: //doi.org/10.1515/crelle-2012-0045.

KM94 M. Kontsevich and Yu. Manin, Gromov-Witten classes, quantum cohomology, and enumerative geometry, Comm. Math. Phys. 164 (1994), no. 3, 525-562; https://doi.org/10.1007/ BF02101490.

Knu08 A. Knutson, Schubert patches degenerate to subword complexes, Transform. Groups 13 (2008), no. 3-4, 715-726; https://doi.org/10.1007/s00031-008-9013-1.

Knu10 _ Puzzles, Positroid varieties, and equivariant K-theory of Grassmannians, 2010, arXiv:1008.4302.

KT03 A. Knutson and T. Tao, Puzzles and (equivariant) cohomology of Grassmannians, Duke Math. J. 119 (2003), no. 2, 221-260; https://doi.org/10.1215/S0012-7094-03-11922-5.

Kum02 S. Kumar, Kac-Moody groups, their flag varieties and representation theory, Progress in Math., vol. 204 (Birkhäuser Boston, Inc., Boston, MA, 2002); https://doi.org/10.1007/ 978-1-4612-0105-2.

Lee04 Y.-P. Lee, Quantum K-theory. I. Foundations, Duke Math. J. 121 (2004), no. 3, 389-424; https://doi.org/10.1215/S0012-7094-04-12131-1.

LM06 C. Lenart and T. Maeno, Quantum Grothendieck polynomials, 2006, arXiv:math.CO/0608232.

LP04 Y.-P. Lee and R. Pandharipande, A reconstruction theorem in quantum cohomology and quantum K-theory, Amer. J. Math. 126 (2004), no. 6, 1367-1379; https://doi.org/10.1353/ajm. 2004.0049.

LP07 C. Lenart and A. Postnikov, Affine Weyl groups in K-theory and representation theory, Int. Math. Res. Not. 2007 (2007), no. 12, rnm038; https://doi.org/10.1093/imrn/rnm038.

Mih06a L. C. Mihalcea, Equivariant quantum Schubert calculus, Adv. Math. 203 (2006), no. 1, 1-33; https://doi.org/10.1016/j.aim.2005.04.002.

Mih06b_, Positivity in equivariant quantum Schubert calculus, Amer. J. Math. 128 (2006), no. 3, 787-803; https://doi.org/10.1353/ajm.2006.0026. 


\section{A Chevalley formula for equivariant quantum $K$-Theory}

Mih07

On equivariant quantum cohomology of homogeneous spaces: Chevalley formulae and algorithms, Duke Math. J. 140 (2007), no. 2, 321-350; https://doi.org/10.1215/ S0012-7094-07-14024-9.

MS99 A.I. Molev and B.E. Sagan, A Littlewood-Richardson rule for factorial Schur functions, Trans. Amer. Math. Soc. 351 (1999), no. 11, 4429-4443; https://doi.org/10.1090/ S0002-9947-99-02381-8.

Oko96 A. Okounkov, Quantum immanants and higher Capelli identities, Transform. Groups 1 (1996), no. 1-2, 99-126; https://doi.org/10.1007/BF02587738.

OO98 A. Okounkov and G. Olshanski, Shifted Schur functions, St. Petersburg Math. J. 9 (1998), no. 2, 239-300.

Per07 N. Perrin, Small resolutions of minuscule Schubert varieties, Compos. Math. 143 (2007), no. 5, 1255-1312; https://doi.org/10.1112/S0010437X07002734.

Pro84 R.A. Proctor, Bruhat lattices, plane partition generating functions, and minuscule representations, European J. Combin. 5 (1984), no. 4, 331-350; https://doi.org/10.1016/ S0195-6698(84)80037-2.

PY17 O. Pechenik and A. Yong, Equivariant K-theory of Grassmannians, Compos. Math. 153 (2017), no. 4, 667-677; https://doi.org/10.1112/S0010437X16008186.

Ric92 R. W. Richardson, Intersections of double cosets in algebraic groups, Indag. Math. (N.S.) 3 (1992), no. 1, 69-77; https://doi.org/10.1016/0019-3577 (92)90028-J.

RT94 Y. Ruan and G. Tian, A mathematical theory of quantum cohomology, Math. Res. Lett. 1 (1994), no. 2, 269-278; https://doi.org/10.4310/MRL.1994.v1.n2.a15.

Ste96 J.R. Stembridge, On the fully commutative elements of Coxeter groups, J. Algebraic Combin. 5 (1996), no. 4, 353-385; https://doi.org/10.1023/A:1022452717148.

Tai13 K. Taipale, K-theoretic J-functions of type A flag varieties, Int. Math. Res. Not. 2013 (2013), no. 16, 3647-3677; https://doi.org/10.1093/imrn/rns156.

Wil06 M. Willems, $K$-théorie équivariante des tours de Bott. Application à la structure multiplicative de la K-théorie équivariante des variétés de drapeaux, Duke Math. J. 132 (2006), no. 2, 271309; https://doi.org/10.1215/S0012-7094-06-13223-4.

Wit95 E. Witten, The Verlinde algebra and the cohomology of the Grassmannian, Geometry, Topology, \& Physics, Conf. Proc. Lecture Notes Geom. Topology, IV (Int. Press, Cambridge, MA, 1995), 357-422.

Anders S. Buch asbuch@math.rutgers.edu

Department of Mathematics, Rutgers University, 110 Frelinghuysen Road, Piscataway, NJ 08854, USA

Pierre-Emmanuel Chaput pierre-emmanuel.chaput@univ-lorraine.fr

Domaine Scientifique Victor Grignard, 239, Boulevard des Aiguillettes, Université de Lorraine, B.P. 70239, F-54506 Vandoeuvre-lès-Nancy Cedex, France

Leonardo C. Mihalcea Imihalce@math.vt.edu

Department of Mathematics, Virginia Tech University, 460 McBryde, Blacksburg VA 24060, USA

Nicolas Perrin nicolas. perrin@uvsq.fr

Laboratoire de Mathématiques de Versailles, UVSQ, CNRS, Université Paris-Saclay, 78035 Versailles, France 